\title{
Is Spatially Integrated Entropy Production Useful to Predict the Dynamics of Ecosystems?
}

\author{
Juan C. Quijano ${ }^{\mathrm{a}}$, Henry Lin ${ }^{\mathrm{a}, *}$ \\ ${ }^{a}$ The Pennsylvania State University, 116 Agricultural Sciences and Industries Building, \\ University Park, PA 16802
}

\begin{abstract}
Production of entropy by macroscopic systems in far from equilibrium as estimated from the spatial integration of the entropy production density have been considered as an important concept that could help understand and predict the evolution of open systems. In this study we have used an extensive dataset of energy fluxes recorded in Ameriflux sites to compute the entropy production across different climates and ecosystems in order to analyze whether there are clear patterns showing a higher entropy production in more vegetated sites or in more developed stages. The entropy budget has been defined over a conceptualized system defined by the Earth Critical Zone. Based on previous studies we have recognized four different formulations for the estimation of entropy production in macroscopic systems: (i) a total entropy production that accounts for all the energy fluxes including radiation fluxes, (ii) an entropy production that includes only those fluxes of energy absorbed within the open system, (iii) an entropy production that includes only living systems and accounts for the biochemical energy absorbed during photosynthesis and its further dissipation in the form of heat, and (iv) an entropy production that includes only incoming and outgoing heat fluxes into the open system that occurs at different temperatures. We quantified these entropy productions at different sites and stages of ecosystem development using available information recorded in 36 Ameriflux sites. We observed rather different magnitudes and patterns in all these formulations of the entropy productions. Therefore, these formulations can not be compared or considered as the same concept as has been done previously because they represent different scales and processes. In addition, we did not find a clear
\end{abstract}

${ }^{*}$ Corresponding author. Tel.: +1 814-865-6726, henrylin@psu.edu 
evidence of a maximum entropy production in more vegetated sites and at more mature stages of development, for any of these four entropy production formulations. These results suggests that hypotheses based on a maximum entropy production estimated from spatial integration over macroscopic systems in far from equilibrium should not be considered as a fundamental principle to understand or predict the evolution of these systems.

Keywords: Entropy production, Critical Zone, Thermodynamics

\section{Introduction}

Since thermodynamic entropy (hereafter entropy) was proposed initially by Clausius in 1865 it has been considered as a promising concept that could lead us to the understanding of the evolution of different kind of open systems. In particular, the production of entropy has been considered as a major concept that could provide important information of a system and predict its behavior. Today, more than two centuries after it was developed many different hypotheses have been proposed in the light of entropy production. However, none of these hypotheses have been fully proven. In particular, a major difficulty lies in the implementation of entropy related principles to areas that are dealing with macroscopic scales where different assumptions including temporal thermal equilibrium and spatial integration of entropy production are considered. In this study we analyze the production of entropy in the Earth Critical Zone, and in order to compute the entropy production over this domain we use an extensive database of energy fluxes that allow us to compare the entropy production across different climates and at different stages of ecosystem development.

The most important hypotheses defined in the light of entropy production are the minimum entropy production principle (MinEnt) by Prigogine (1947) and the maximum entropy production principle (MEPP) proposed initially by Ziegler $(1963,1983)$. On the one hand, MinEnt suggests that systems in the linear non-equilibrium regime reach a steady state at which the entropy production from the system is constant and attains a minimum value (Kondepudi and Prigogine, 1998). Previous studies have questioned the validity of MinEnt (Martyushev and Seleznev, 2006; Martyushev, 2013; Jaynes, 1980; Palffy-Muhoray, 2001; Danielewicz-Ferchmin and Ferchmin, 2000; Hoover, 2002). In particular, Martyushev and Seleznev (2006); Martyushev (2013) showed that MinEnt could be conceptualized as an special case of MEPP. 
On the other hand, MEPP as proposed initially by Ziegler (1983) suggests that when thermodynamic forces are preset the fluxes are such that there is a maximization of the entropy production density (Martyushev and Seleznev, 2006). It is important to keep in mind that MEPP implies a maximization of the entropy density $\delta$ defined over a fundamental spatial domain where the thermal equilibrium assumption remains valid. Different fields, including atmospheric sciences, hydrology, ecology, pedology, and geomorphology, have looked at MEPP as an alternative to predict the evolution of systems. However, these fields deal with macroscopic domains where the definition of such a fundamental scale is challenging. As a result, previous studies (Lucia, 2014; Ozawa et al., 2003; Kleidon et al., 2010) in these fields have modified the MEPP. This modified version, here we call ModMEPP, suggests that evolution of open macroscopic systems in far from equilibrium conditions is guided toward a maximum entropy production $\sigma$ that is defined over the entire domain by spatial integration of the entropy production density:

$$
\sigma=\int \delta
$$

where $\delta$ is the entropy production density. This formulation involves an integration of the entropy production density over domains where the thermal equilibrium assumption is not longer valid. Therefore, some previous authors such as Martyushev (2013) claim that maximization of $\sigma$ as suggested in ModMEPP may not be valid.

Although the validity of ModMEPP is still under debate, it could have a significant contribution in areas dealing with macroscopic systems. Computation of $\sigma$ by spatial integration could be attained with measurements of energy fluxes and temperature performed in macroscopic domains. Different examples of macroscopic systems where such an approach has been performed to compute the entropy production include a cell (Lucia, 2014), a leaf (Aoki, 1989), an ecosystem (Aoki, 1995; Holdaway et al., 2010), the global water cycling (Porada et al., 2011; Westhoff et al., 2014), a portion of the atmosphere (Paltridge, 1975; Pauluis and Held, 2002), or even the entire planet (Ichiro, 1983-03-15; Lorenz, 2002). As a result validation of ModMEPP is more doable than MEPP. In fact, while there are still no clear methodologies to validate MEPP, many previous studies have performed validations of ModMEPP. For instance, in atmospheric sciences different studies trying to understand the exchange of heat fluxes within the atmosphere and to the ocean have found that ModMEPP is able to reproduce observed pat- 
terns (see Ozawa et al. (2003)). Similarly studies with FLUXNET towers in ecosystems found that entropy production increases in the more developed ecosystems supporting ModMEPP (Holdaway et al., 2010). However, more recent studies in ecological networks (Meysman et al., 2010) claimed that the evolution of such systems is not always guided by a maximum production of entropy. In addition, studies performed on different atmospheric conditions show that rather different conditions and dissipation of energy could attain the same magnitude of entropy production by different mechanisms (Pauluis and Held, 2002), which indirectly disprove ModMEPP.

ModMEPP has also been proposed as a leading hypothesis to understand the evolution of terrestrial ecosystems. Previous studies have suggested a connection between life and higher production of entropy (Schneider and Kay, 1994; Ulanowicz and Hannon, 1987), and according to Kleidon (2004) the presence of vegetation in different parts of the Earth support this hypothesis. In addition, Holdaway et al. (2010) compared $\sigma$ at different stages of ecosystem development and found that $\sigma$ increases in the more developed ecosystems, which in turn support ModMEPP. However, some of previous studies have been supported on deductions based on expected patterns, instead of actual quantifications of $\sigma$. On the other hand, the studies that have quantified $\sigma$ have analyzed few ecosystems and have used different periods throughout the year that has been selected arbitrarily. Therefore, it is important to perform a more comprehensive comparison, that includes different ecosystems and climates. In this study we used an extensive database of energy fluxes over different sites spanning climates, to bring more evidence into the patterns of the entropy production $\sigma$ in terrestrial ecosystems, and in particular analyze whether these patterns support ModMEPP. The system under study is the Earth Critical Zone (CZ), which according to NRC (2001), is defined as the heterogeneous, near surface environment in which complex interactions involving rock, soil, water, air, and living organisms regulate the natural habitat and determine the availability of life-sustaining resources. The definition of this system allows us to take advantage of the extensive dataset recorded in FLUXNET Towers (Ameriflux Sites) to analyze the budget of entropy in different sites and at different developmental stages.

An important consideration that most studies have overlooked is the actual formulation that is implemented for the estimation of the entropy production. Previous studies have used different formulations for $\sigma$, and the outcomes from different formulations may result in different patterns. Based 
on previous studies we recognized the next formulations that have been considered for the estimation of $\sigma$ : (i) some approaches include all the sources of energy that reach the system under analysis, including the incoming radiation, heat and chemical energy, (ii) other approaches neglect radiation fluxes because they claim most of radiation is dissipated instantaneously by the surface and include only the energy fluxes in the form of heat and chemical energy that goes into the system of analysis, and (iii) other approaches consider that the most interesting component to analyze are the fluxes with the highest levels of specific exergy and include only the chemical energy (neglecting heat) that is absorbed by the system under analysis. These different formulations for the estimation of $\sigma$ are defined on different scales and included different processes. Thus, it is unclear whether all of them lead to similar patterns, and even more if all of them support ModMEPP.

The main goals of this study are: (i) to quantify the production of entropy $\sigma$ in the $\mathrm{CZ}$ for the formulations of entropy production that have been discussed above, (ii) to analyze whether computed $\sigma$ from these formulations have the same patterns across climates and stages of development, and (iii) to analyze whether these patterns support ModMEPP as a leading hypothesis for terrestrial ecosystems.

\section{Theory}

\subsection{Formulations for Entropy Production}

Previous studies have implemented different formulations for the computation of entropy. In this section we define these formulations and explain how these approaches are applied to the $\mathrm{CZ}$.

\subsubsection{Total Entropy Production $\sigma$}

Previous studies (Aoki, 1987; Holdaway et al., 2010) have conceptualized the production of entropy by considering all the fluxes that reach the domain under analysis. As a result, the production of entropy in these approaches is quantified by including the exchanges of radiation, heat, and chemical entropy fluxes with the environment.

In order to analyze the budget of entropy and energy in the $\mathrm{CZ}$ it is important to define the boundaries that delineate the CZ. According to Kleidon et al. (2012) and Quijano and Lin (2014), the lower boundaries of the $\mathrm{CZ}$ are defined by the bottom of the regolith, and the upper boundary as an imaginary limit right above the canopy. In this study we use the same 
conceptualization (Figure 1). The delineation of the lateral boundaries associated with the $\mathrm{CZ}$ is more subjective. They may range from a soil profile, an ecosystem, to a geographic cell, a watershed, or an entire continent (Quijano and Lin, 2014). In this study the spatial delineation of the CZ is given by the ecosystem scale defined by the footprint of the FLUXNET tower. Figure 1 shows a schematic representation of the control volume used for the $\mathrm{CZ}$ in this study. Neglecting energy fluxes due to mass transport, and horizontal and bottom fluxes of heat (these fluxes are significantly lower compared with the other fluxes in Equation 2) (Quijano and Lin, 2014), the energy balance of the control volume as defined in Figure 1 is given by:

$$
\frac{d E_{c z}}{d t}=E_{S W, \text { in }}-E_{S W, o u t}+E_{L W, \text { in }}-E_{L W, \text { out }}-E_{L E}-E_{H}
$$

where $\mathrm{dE}_{\mathrm{cz}}$ is the rate of change of energy within the $\mathrm{CZ}$ per unit area, $\mathrm{E}_{\mathrm{SW}}, \mathrm{E}_{\mathrm{LW}}, \mathrm{E}_{\mathrm{LE}}$, and $\mathrm{E}_{\mathrm{H}}$ are the fluxes of energy related to shortwave (SW) radiation, longwave $(\mathrm{LW})$ radiation, latent heat $(\mathrm{LE})$, and sensible heat $(\mathrm{H})$ fluxes, respectively. Using the same formulation we can define the production of entropy by the $\mathrm{CZ}$ as:

$$
\sigma_{\mathrm{cz}}=\frac{\mathrm{dS}_{\mathrm{cz}}}{\mathrm{dt}}+\left(\mathrm{S}_{\mathrm{SW}, \text { out }}-\mathrm{S}_{\mathrm{SW}, \text { in }}+\mathrm{S}_{\mathrm{LW}, \text { out }}-\mathrm{S}_{\mathrm{LW}, \text { in }}+\mathrm{S}_{\mathrm{LE}}+\mathrm{S}_{\mathrm{H}}\right) \geq 0
$$

where $d S_{c z}$ is the rate of change of entropy within the CZ per unit area, $S$ refers to fluxes of entropy associated with the different terms in Equation 2, and $\sigma_{c z}$ is the total production of entropy by the CZ.

\subsubsection{Internal Production of Entropy $\sigma_{i n t}$}

A significant fraction of the radiation that is coming to the surface is dissipated instantaneously. As a result, the fluxes of radiation and heat used in this dissipation are significantly higher than the fluxes of energy that are absorbed by the surface. As a result, previous studies in Earth Sciences (Rasmussen et al., 2011) have neglected the fluxes of radiation and heat dissipated by the surface, and consider only the fluxes of energy, entropy, 
and exergy that are absorbed by the system under analysis. The rationale is that fluxes absorbed by the system are directly responsible for the alteration of the internal structure of it and therefore will provide more explanation for its evolution.

The CZ is experiencing a continuous exchange of radiation fluxes with its environment. However, incoming radiation (SW and LW) is dissipated instantaneously by the surface in the form of outgoing radiation (SW and LW), $\mathrm{LE}$ and $\mathrm{H}$ heat. The fraction of energy that is absorbed by the $\mathrm{CZ}$ is mostly in the form of heat and chemical components, in particular photosynthetic compounds. Considering only photosynthetic compounds and heat, we can express the energy balance in the $\mathrm{CZ}$ as:

$$
\frac{\mathrm{dE}_{\mathrm{cz}}}{\mathrm{dt}}=\mathrm{E}_{\mathrm{SW}, \mathrm{pho}}+\mathrm{E}_{\mathrm{Q}, \mathrm{G}}+\mathrm{E}_{\mathrm{Q}, \mathrm{A}}
$$

where $\mathrm{E}_{\mathrm{SW} \text {,pho }}$ refers to the $\mathrm{SW}$ radiation flux that is captured through photosynthesis, and $\mathrm{E}_{\mathrm{Q}, \mathrm{G}}+\mathrm{E}_{\mathrm{Q}, \mathrm{A}}$ are the net heat fluxes to the below and aboveground regions of the $\mathrm{CZ}$, respectively. Both $\mathrm{E}_{\mathrm{Q}, \mathrm{G}}$ and $\mathrm{E}_{\mathrm{Q}, \mathrm{A}}$ can be decomposed in incoming and outgoing fluxes of heat. Also, note that some fraction of the outgoing heat fluxes come from the dissipation of work and respiration of living systems within the $\mathrm{CZ}\left(\mathrm{E}_{\mathrm{Q}, \mathrm{resp}}\right)$, thus we can express Equation 4 as:

$$
\begin{gathered}
\frac{\mathrm{dE}_{\mathrm{cz}}}{\mathrm{dt}}=\underbrace{\mathrm{E}_{\mathrm{SW}, \text { pho }}-\mathrm{E}_{\mathrm{Q}, \text { resp }}}_{\text {Biochemical }\left(\mathrm{E}_{\text {bio }}\right)}+ \\
\underbrace{\underbrace{\mathrm{E}_{\mathrm{Q}, \mathrm{G}, \text { in }}-\mathrm{E}_{\mathrm{Q}, \mathrm{G}, \text { out }}}_{\text {Below-ground }}+\underbrace{\mathrm{E}_{\mathrm{Q}, \mathrm{A}, \text { in }}-\mathrm{E}_{\mathrm{Q}, \mathrm{A}, \text { out }}}_{\text {Above-ground }}+\mathrm{E}_{\mathrm{Q}, \text { resp }}}_{\text {Heat }\left(\mathrm{E}_{\mathrm{Q}}\right)},
\end{gathered}
$$

where the energy into the $\mathrm{CZ}$ is divided into two main components, (i) net biochemical $\left(\mathrm{E}_{\mathrm{bio}}\right)$ fluxes that refer to the uptake of $\mathrm{SW}$ radiation by photosynthesis and its subsequently dissipation in the form of heat after respiration and work, and (ii) net heat $\left(\mathrm{E}_{\mathrm{Q}}\right)$ fluxes that refer to incoming and ougoing fluxes of heat energy in the $\mathrm{CZ}$.

Based on the energy balance in Equation 5 it is possible to define the production of entropy that includes only the fluxes of energy that are absorbed by the CZ, as: 


$$
\begin{aligned}
\sigma_{\text {cz,int }}=\frac{\mathrm{dS} \mathrm{cz}_{\mathrm{cz}}}{\mathrm{dt}} & +\underbrace{\left(\mathrm{S}_{\mathrm{Q}, \text { resp }}-\mathrm{S}_{\mathrm{SW}, \text { pho }}\right)}_{\sigma_{\text {bio }}} \\
+ & \underbrace{\left(\mathrm{S}_{\mathrm{Q}, \mathrm{A}, \text { out }}-\mathrm{S}_{\mathrm{Q}, \mathrm{A}, \text { in }}\right)+\left(\mathrm{S}_{\mathrm{Q}, \mathrm{G}, \text { out }}-\mathrm{S}_{\mathrm{Q}, \mathrm{G}, \text { in }}\right)-\mathrm{S}_{\mathrm{Q}, \text { resp }}}_{\sigma_{\mathrm{Q}}} \geq 0
\end{aligned}
$$

where S refers to exchange fluxes of entropy, and $\sigma_{\mathrm{cz}, \text { int }}$ is the instantaneous production of entropy within the CZ. According to Equation 6 the internal production of entropy in a system delineating the CZ has two main components: (i) $\sigma_{b i o}$, which is the entropy produced by the transformation of SW radiation to chemical energy stored in reduced carbon compounds, and its further conversion to heat (after oxidation and work performed), and (ii) $\sigma_{Q}$, which is the entropy produced by fluxes of heat that enter and leave the system at different temperatures.

In addition, note that $\sigma_{\mathrm{cz}, \text { int }}$ is embedded in $\sigma_{\mathrm{cz}}$, and:

$$
\sigma_{\mathrm{cz}}=\sigma_{\mathrm{cz}, \mathrm{int}}+\sigma_{\mathrm{rad}}
$$

where $\sigma_{\text {rad }}$ is the production of entropy caused by the instantaneous transformation of SW radiation at the surface.

\subsubsection{Biological Entropy Production $\sigma_{\text {bio }}$}

Previous studies (Meysman et al., 2010; Bendoricchio and Jorgensen, 1997) have considered only the processes that are directly connected with living systems. These approaches include only the fluxes of energy, entropy, and exergy that are associated with the reduction and oxidation of organic compounds. According to these studies, the exergy that is stored in living systems is by far the most important source of exergy stored in ecosystems. Also, this production of entropy is important because it is directly related to the work performed by living systems, and this work is the base of ecological interactions and ecosystem dynamics. The production of entropy associated with living systems is originated by the oxidation of photosynthetic products with low entropy (high exergy), and subsequently dissipation of heat with higher entropy (lower exergy). Note that the definition of $\sigma_{\text {bio }}$ presented in Equation 6 represents the production of entropy by considering only biological systems as in previous studies. 


\subsection{Annual Production of Entropy}

In previous section we defined different formulations for the instantaneous production of entropy, $\sigma_{\mathrm{cz}}, \sigma_{\mathrm{cz}, \mathrm{rad}}, \sigma_{\mathrm{cz}, \text { int }}, \sigma_{\mathrm{Q}}$, and $\sigma_{\mathrm{pho}}$, in the $\mathrm{CZ}$ based on different processes that are included. It is worth mentioning that these definitions refer to entropy production in a conceptualized macroscopic system and already involve a spatial integration. Now, if we integrate these instantaneous productions of entropy over a year we obtain the total entropy produced in a year, which is a more fair quantity to compare the productions of entropy over different ecosystems:

$$
\begin{aligned}
& \eta_{\mathrm{cz}}=\int_{\text {year }} \frac{\mathrm{d} \mathrm{S}_{\mathrm{cz}}}{\mathrm{dt}}+\underbrace{}_{\eta_{\mathrm{SW}}} \underbrace{\underbrace{}_{\text {year }}}_{\eta_{\mathrm{LW}} \mathrm{S}_{\mathrm{SW}, \text { out }}-\mathrm{S}_{\mathrm{SW}, \text { in }}}+ \\
& \underbrace{\int_{\mathrm{LW}, \text { out }}-\mathrm{S}_{\mathrm{LW}, \text { in }}}_{\text {year }}+\underbrace{\int_{\text {year }} \mathrm{S}_{\mathrm{LE}}}_{\eta_{\mathrm{LE}}}+\underbrace{\int_{\text {year }} \mathrm{S}_{\mathrm{H}} \geq 0}_{\eta_{\mathrm{H}}}
\end{aligned}
$$

Term $\eta_{\mathrm{cz}}$ is the total production of entropy by the CZ in a year. Terms $\eta_{\mathrm{SW}}$, $\eta_{\mathrm{LW}}, \eta_{\mathrm{LE}}$, and $\eta_{\mathrm{H}}$ refer to the different components that are associated with SW and LW radiation and LE and $\mathrm{H}$ heat over a year, respectively. Similarly:

$$
\begin{aligned}
& \eta_{\text {cz,int }}=\underbrace{\int_{\text {year }} \mathrm{S}_{\mathrm{Q}, \text { resp }}-\mathrm{S}_{\mathrm{SW}, \text { pho }}+\frac{\mathrm{d \textrm {S } _ { \mathrm { cz } }}}{\mathrm{dt}}}_{\eta_{\text {bio }}} \\
& +\underbrace{\int_{\text {year }} S_{Q, \mathrm{G}, \text { out }}-\mathrm{S}_{\mathrm{Q}, \mathrm{G}, \text { in }}+\mathrm{S}_{\mathrm{Q}, \mathrm{A}, \text { out }}-\mathrm{S}_{\mathrm{Q}, \mathrm{A}, \text { in }}-\mathrm{S}_{\mathrm{Q}, \text { resp }}}_{\eta_{Q}} \geq 0 .
\end{aligned}
$$

where $\eta_{\mathrm{cz}, \text { int }}$ refers to the total production of entropy within the CZ in a year. Terms $\eta_{\text {bio }}$ and $\eta_{\mathrm{Q}}$ refer to the components associated with biological systems, and heat fluxes, respectively. 
At the annual scale the storage of energy, entropy, and exergy associated with heat fluxes is negligible (Rasmussen et al., 2011), thus at the annual scale the changes in energy and entropy are linked mostly to biomass accumulation, thus:

$$
\begin{aligned}
& \left.\left.\mathrm{dE}_{\mathrm{cz}}\right|_{\text {year }} \approx\left(\mathrm{E}_{\mathrm{SW}, \text { pho }}-\mathrm{E}_{\mathrm{Q}, \text { resp }}\right)\right|_{\text {year }} \\
& \left.\left.\mathrm{dS}_{\mathrm{cz}}\right|_{\text {year }} \approx\left(\mathrm{S}_{\mathrm{SW} \text {,pho }}-\mathrm{S}_{\mathrm{Q}, \text { resp }}\right)\right|_{\text {year }} .
\end{aligned}
$$

Therefore, $\frac{\mathrm{dS}}{\mathrm{dt}}$ is considered as part of $\eta_{\mathrm{bio}}$ in Equation 9.

\subsection{Exergy Destruction}

Following the Gouy-Stodola theorem (Bejan, 2013; Lucia, 2013), the destruction of exergy by a system can be computed as:

$$
\Gamma \approx \mathrm{T}_{\mathrm{o}} \eta
$$

where $\Gamma$ is the destruction of exergy, and $T_{0}$ is the temperature of a hypothetical reference environment to the system. From Equation 11 it is possible to define the destruction of exergy $\Gamma_{\mathrm{cz}}, \Gamma_{\mathrm{cz}, \mathrm{rad}}, \Gamma_{\mathrm{cz}, \mathrm{int}}, \Gamma_{\mathrm{bio}}$, and $\Gamma_{\mathrm{Q}}$ associated with the different productions of entropy that have been described above, $\eta_{\mathrm{cz}}, \eta_{\mathrm{cz}, \mathrm{rad}}, \eta_{\mathrm{cz}, \mathrm{int}}, \eta_{\mathrm{bio}}$, and $\eta_{\mathrm{Q}}$.

However, note that a hypothetical environment for the CZ could be represented by rather different systems such as the atmosphere, a nearby CZ, the lithosphere, or even the sun (Jørgensen et al., 2011; Quijano and Lin, 2014). Therefore, estimation of the reference temperature of a hypothetical environment is challenging for the CZ. Although a direct quantification of exergy destruction is not possible, we see from Equation 11 that the exergy destruction is proportional to the entropy production, and as a result can be associated with different processes in the CZ.

\section{Methods}

\subsection{Sites}

In this study we have selected 36 Ameriflux sites with available information to compute $\sigma_{\mathrm{cz}}$ or $\sigma_{\mathrm{cz}, \text { int }}$. Table 1 shows the complete list of selected 
sites, and Figure 2 shows the location of all these sites. In addition, the Online Supplement Section 1 shows the specific years of information that were utilized for each of the figures generated in this study.

\subsection{Entropy Calculation with FLUXNET data}

In this section we introduce the formulations to compute the production of entropy from information recorded in FLUXNET towers. These equations are based on previous approaches such as Aoki (1987, 1989), Holdaway et al. (2010), and Quijano and Lin (2014). The entropy flux associated with an energy flux of SW radiation $\left(\mathrm{E}_{\mathrm{SW}}\right)$ recorded in a FLUXNET tower is given by:

$$
\mathrm{S}_{\mathrm{SW}}=\mathrm{RF}_{\mathrm{SW}, \mathrm{dir}}\left(\frac{\mathrm{E}_{\mathrm{SW}, \mathrm{dir}}}{\mathrm{T}_{\mathrm{sun}}}\right)+\mathrm{RF}_{\mathrm{SW}, \mathrm{dif}}\left(\frac{\mathrm{E}_{\mathrm{SW}, \mathrm{dif}}}{\mathrm{T}_{\mathrm{sun}}}\right)
$$

where $\mathrm{E}_{\mathrm{SW} \text {,dir }}$ and $\mathrm{E}_{\mathrm{SW} \text {,dif }}$ are the fluxes of direct and diffuse $\mathrm{SW}$ radiation, respectively. The terms $R F_{S W, d i r}$ and $R F_{S W, d i f}$ are the radiation factors $(R F)$ for direct and diffuse SW radiation, respectively. Radiation factors are computed based on Wu and Liu (2010) and Wright et al. (2001), see Online Supplement Section 2. Term $\mathrm{T}_{\text {sun }}$ is the temperature of the Sun (Table 2). Similarly, the entropy flux associated with LW radiation recorded in a FLUXNET tower is given by:

$$
\mathrm{S}_{\mathrm{LW}}=\mathrm{RF}_{\mathrm{LW}}\left(\frac{\mathrm{E}_{\mathrm{LW}}}{\mathrm{T}_{\mathrm{emi}}}\right)
$$

where $\mathrm{RF}_{\mathrm{LW}}$ is the radiation factor for LW radiation (see Online Supplement Section 2), and $\mathrm{T}_{\mathrm{emi}}$ is the temperature of emission associated with the LW radiation. For incoming $L W$ radiation $T_{\text {emi }}$ is the atmospheric temperature, while for outgoing $L W$ radiation $T_{\text {emi }}$ is an effective temperature of the $\mathrm{CZ}$ $\left(\mathrm{T}_{\mathrm{cz}}\right)$ that is computed as:

$$
\mathrm{T}_{\mathrm{cz}}=\left(\frac{\mathrm{E}_{\mathrm{LW}, \mathrm{out}}}{\varepsilon \lambda}\right)^{1 / 4}
$$

where $\lambda$ is the Stefan-Boltzmann constant, and $\varepsilon$ is an effective emissivity of the CZ. The entropy flux due to fluxes of LE and $\mathrm{H}$ from the $\mathrm{CZ}$ are computed as:

$$
\mathrm{S}_{\mathrm{LE}}=\left(\frac{\mathrm{E}_{\mathrm{LE}}}{\mathrm{T}_{\mathrm{cz}}}\right) \quad \mathrm{S}_{\mathrm{H}}=\left(\frac{\mathrm{E}_{\mathrm{H}}}{\mathrm{T}_{\mathrm{cz}}}\right) .
$$


The flux of energy in the form of photosynthesis captured by the CZ is computed as:

$$
\mathrm{E}_{\mathrm{SW}, \text { pho }}=h_{\mathrm{BIO}} \mathrm{GPP}
$$

where GPP is the Gross Primary Production provided by the Ameriflux network, and $h_{\mathrm{BIO}}$ is the specific biomass enthalpy (Nikolov, 1995; Rasmussen et al., 2011). Using a similar approach we can compute the flux of energy that is released as heat after respiration.

$$
\mathrm{E}_{\mathrm{Q}, \text { resp }}=h_{\mathrm{BIO}} \text { Reco }
$$

where Reco is the net flux of ecosystem respiration. As mentioned above at the annual scale the rate of energy change in the CZ is mostly associated with biomass (Equation 10). Therefore, the rate of change of entropy is defined based only on photosynthetic and respiration fluxes, as:

$$
\mathrm{dS}_{\mathrm{cz}}= \begin{cases}\left(\mathrm{dE}_{\mathrm{cz}, \mathrm{pho}}\right) \xi_{\mathrm{sW}, \mathrm{in}, \mathrm{yr}}, & \text { if } \mathrm{dE}_{\mathrm{cz}, \mathrm{pho}} \neq 0 \\ 0, & \text { if } \mathrm{dE}_{\mathrm{cz}, \mathrm{pho}}=0\end{cases}
$$

where $\xi_{\mathrm{sw} \text {,in,yr }}$ is a mean annual ratio between the entropy and energy fluxes of the incoming SW radiation that is captured through photosynthesis in the CZ. In other words, we assume that the biomass that is stored, has on average, a ratio of entropy to energy equal to $\xi_{\mathrm{SW}, \mathrm{in}, \mathrm{yr}}$, which is given by:

$$
\xi_{\mathrm{SW}, \text { in,yr }}=\frac{\int_{\text {year }} S_{\mathrm{SW}, \text { in }}}{\int_{\text {year }} E_{\mathrm{SW}, \text { in }}} .
$$

The net flux of heat into the $\mathrm{CZ}$ is computed as:

$$
\mathrm{E}_{\mathrm{Q}}=\mathrm{Rn}-\mathrm{E}_{\mathrm{LE}}-\mathrm{E}_{\mathrm{H}}-\mathrm{E}_{\mathrm{SW}, \text { pho }}+\mathrm{E}_{\mathrm{Q}, \mathrm{resp}}
$$

where $\mathrm{Rn}$ is the net radiation recorded by the FLUXNET tower. If the ground heat flux $\mathrm{E}_{\mathrm{Q}, \mathrm{G}}$ is recorded by the tower it is possible to differentiate the below- $\left(\mathrm{E}_{\mathrm{Q}, \mathrm{G}}\right)$ and above-ground $\left(\mathrm{E}_{\mathrm{Q}, \mathrm{A}}\right)$ fluxes of heat. The entropy production associated with heat fluxes absorbed by the $\mathrm{CZ}$ is then given by: 


$$
\eta_{\mathrm{Q}}=\frac{\mathrm{E}_{\mathrm{Q}}}{\mathrm{T}_{\mathrm{cz}}}
$$

Previous studies have reported lack of energy balance closure in most of the FLUXNET towers (Fisher et al., 2007; Oliphant et al., 2004; Wilson et al., 2002). In turn, this may impact the computation of $\mathrm{E}_{\mathrm{Q}}$. In this study we implement an additional correction to $\mathrm{E}_{\mathrm{Q}}$ in order to account for this closure. Online Supplement Section 3 shows this correction in more detail.

\section{Results}

\subsection{Total Entropy Production by the Critical Zone $\sigma_{c z}$}

Figure 3a shows a plot of $\eta_{\mathrm{cz}}$ vs $\mathrm{E}_{\mathrm{SW} \text {,in }}$ for 24 different sites with available information to compute $\eta_{c z}$ for at least a year. There is a clear pattern showing that $\eta_{\mathrm{Cz}}$ increases with $\mathrm{E}_{\mathrm{SW} \text {,in }}$. These results support the findings obtained by Aoki $(1987,1989)$ who found a linear relationship between the incoming global radiation and the production of entropy in plant leaves. However, here these results are obtained for the entire CZ (See Figure 1) and for many sites in different climates. This pattern is expected as SW radiation is the source of incoming energy with the lowest entropy. Thus, a higher flux of SW radiation would increase the production of entropy.

Surface albedo $(\alpha)$ directly measures the fraction of incoming SW radiation that is transformed to other forms of energy. Therefore, when $\alpha$ is included in Figure 3a it helps to see a clearer representation of the trends that are observed for $\eta_{\mathrm{cz}}$. Although both $\mathrm{E}_{\mathrm{SW} \text {,in }}$ and $\alpha$ are able to explain some of the trends observed in $\eta_{c z}$ in different sites, there are some variations that are not completely captured by these two variables (Figure 3). This suggests that spatial variations associated with temperature or emission of $\mathrm{LE}$ or $\mathrm{H}$ heat also play a role in the production of entropy. Figure $3 \mathrm{~b}$ shows a plot of $\eta_{\mathrm{cz}}$ vs the effective temperature of the $\mathrm{CZ}\left(\mathrm{T}_{\mathrm{cz}}\right)$. Contrary to intuition there is a clear trend showing that $\eta_{\mathrm{cz}}$ increases with $\mathrm{T}_{\mathrm{cz}}$. These results also suggests that the total exergy dissipated by the $\mathrm{CZ} \Gamma_{\mathrm{cz}}$ (following Equation 11) increases with $\mathrm{T}_{\mathrm{cz}}$ which is opposite to previous hypotheses claiming that cooler ecosystems will result in higher dissipation (destruction) of exergy (Kay, 2000). As we will show in Section 4.1 .1 a cooler ecosystem could result in higher $\eta_{\mathrm{cz}}$ if all the ecosystems that are compared are exposed to the same climate; however according to Figure $3 \mathrm{~b}$ this pattern does not hold anymore if we compare ecosystems experiencing different climates because the amount 
of incoming SW radiation as well as the atmospheric temperature impact the entropy production.

Figure 4 shows the annual energy budgets and the production of entropy for the 24 sites presented in Figure 3. The left bar displays the incoming energy reaching the $\mathrm{CZ}$ with two main components, SW and LW radiation. The incoming energy in the form of LW radiation is higher than SW radiation in all the sites. The bar in the middle shows the dissipation of energy from the CZ. As expected, the dissipation in the form of LW radiation is the most prominent component. There is an important fraction that is dissipated in the form of LE and $\mathrm{H}$ heat, and this fraction varies across the different sites. The bars on the right in Figure 4 show the total production of entropy in the $\mathrm{CZ}$ with the respective components of the entropy budget according to Equation 8. Note that component $\eta_{\mathrm{SW}}$ is negative in all the sites. This negative value results from a significantly higher incoming flux of SW radiation when compared with the outgoing flux of SW radiation. However, the total production of entropy by the $\mathrm{CZ}$ when all the components are considered is always positive, following the second law of thermodynamics. It can be seen that $\eta_{\mathrm{LW}}$ and $\eta_{\mathrm{LE}}$ are in most of the sites the most important components. As expected, in arid and semi arid climates $\eta_{\mathrm{LE}}$ is significantly lower and the $\eta_{\mathrm{LW}}$ is higher when compared to other climates.

The highest $\eta_{\mathrm{SW}}$ in Figure 4 occurs in sites experiencing semi arid climates (such as sites \#2 and 16 in Table 1). Semi arid sites are able to produce more entropy because there is more incoming SW radiation (less clouds), and in addition there is a preference for LW instead of LE for the dissipation of energy. Note that $R F_{L W}>1$ (see Equation 13) and therefore LW radiation contains higher entropy than heat ( $W u$ and Liu, 2010). Therefore, dissipation of incoming SW radiation in the form of LW radiation instead of $\mathrm{LE}$ or $\mathrm{H}$ heat would enhance the production of entropy. In addition, the gradients in temperature throughout the day are magnified in arid climates enhancing the production of entropy.

\subsubsection{Dynamics of $\eta_{c z}$ Over Time}

Figure 5 shows $\eta_{\mathrm{cz}}$ across different stages of ecosystem development in nine different sites. The value of $\eta_{\mathrm{cz}}$ shown in Figure 5 is the result of an average performed over all historical periods with available records to compute $\eta_{\mathrm{cz}}$ at different stages of development. This approach allows for a fair comparison between the different stages of development. We observe that for all the sites displayed in Figure 5 there is a consistent pattern that shows an inverse 
relationship between $\eta_{\mathrm{cz}}$ and $\alpha$. As $\alpha$ reduces there is a higher $\eta_{\mathrm{cz}}$. This suggests that when ecosystems under the same climate are compared the trends of $\eta_{\mathrm{cz}}$ can be captured only by $\alpha$.

In addition, Figure 5 shows that in most of these sites $\eta_{c z}$ increases with the stage of the ecosystem, and becomes higher as $\mathrm{T}_{\mathrm{cz}}$ reduces (not shown). The same pattern was reported by Holdaway et al. (2010) for different successional stages in the Amazon, and supports ModMEPP. As ecosystems develop there is an increase in the above-ground biomass and leaf area index (LAI) that induces a reduction of $\alpha$. This explains the general pattern observed in different ecosystems with a higher $\eta_{c z}$ as they become more mature. However, in some ecosystems such as in sites \#6, 30, and 36 such a pattern does not hold. The different patterns observed at these three sites could be explained by different causes. In site \#30 the younger site corresponds to a disturbance. This suggests that throughout a disturbance event there could be a transition where $\alpha$ becomes lower and thus increases $\eta_{c z}$. In site \#36 the the LAI in all the three states of development is significantly low and $\alpha$ is controlled by the soil type rather than the vegetation. Finally, in site \#6 the species composition at the different stages of development varies significantly (pines to deciduous), which suggests that transition of species across ecosystem development may cause a reduction (instead of an increase) in $\eta_{\mathrm{cz}}$.

\subsection{Entropy Production Within the Critical Zone $\sigma_{\mathrm{cz}, \mathrm{int}}$}

In this section we examine the production of entropy associated with the internal processes in the CZ $\left(\eta_{\mathrm{cz}, \text { int }}\right)$, and analyze whether it shows a similar trend as $\eta_{\mathrm{cz}}$. We observed that $\eta_{\mathrm{cz}}$ is connected with the total destruction of exergy in the CZ. This destruction in turn is connected to the transformation of SW radiation to other forms of energy that is directly linked to $\alpha$. It has been observed that more developed ecosystems tends to reduce the albedo resulting in a higher $\eta_{c z}$ (Figure 5). Figure 6 shows the fraction of the transformed SW radiation that is actually captured through photosynthesis. It can be seen that this fraction is fairly low $(\approx 2 \%)$. Figure 6 also shows the ratio $\eta_{\mathrm{cz}, \text { int }} / \eta_{\mathrm{cz}}$ for those sites where there is at least one year of information available to compute both $\eta_{\mathrm{cz}}$ and $\eta_{\mathrm{cz}, \text { int }}$. We observe that this ratio is close to $2-3 \%$, which suggests that about $97-98 \%$ of the exergy that is destroyed in the CZ (Equation 11) is due to an instantaneous and irreversible transformation of SW radiation at the top of the $\mathrm{CZ}\left(\sigma_{\mathrm{cz}, \mathrm{rad}}\right)$. Therefore, as ecosystems mature, they reduce $\alpha$ and increases $\sigma_{\mathrm{cz}}$, and they become more effective to destroy exergy. However, only a small fraction of this exergy that 
is destroyed occurs internally to provide work and sustain different processes within the $\mathrm{CZ}$.

Figure 7 shows the energy balance within the $\mathrm{CZ}$ and $\eta_{\mathrm{cz} \text {,int }}$ for 16 sites where there is available information to compute $\eta_{\mathrm{cz}, \text { int }}$ for at least a year. We observe that $\mathrm{E}_{\mathrm{Q}}$ is significant and represents the most prominent source of energy that is absorbed by the CZ. In those sites where information of $\mathrm{E}_{\mathrm{Q}, \mathrm{G}}$ is available we are able to differentiate the fraction of incoming heat fluxes that are related to $\mathrm{E}_{\mathrm{Q}, \mathrm{G}}$ and $\mathrm{E}_{\mathrm{Q}, \mathrm{A}}$. The bars on the right in Figure 7 shows $\eta_{\mathrm{cz}, \text { int }}$ and the respective components following Equation 9. There are important facts that should be highlighted. First, there is an important fraction of $\eta_{\mathrm{cz}, \text { int }}$ that results from the fluxes of heat $\left(\eta_{\mathrm{Q}}\right)$. Although the net annual storage of energy due to heat fluxes is negligible, there are differences in temperature between the incoming and outgoing fluxes that result in production of entropy. In particular, these patterns are more critical in sites experiencing a hot semi arid climate (Bsh) such as site \#24 (Table 1) where the gradients in temperature throughout the day in the $\mathrm{CZ}$ result in a significant $\eta_{\mathrm{Q}}$ (See Figure 8). Second, there is also an important production of entropy associated with biological components $\left(\eta_{\text {bio }}\right)$. Although the incoming fluxes of energy linked to photosynthesis are considerably lower than those of heat, $\eta_{\text {bio }}$ becomes significant because it involves the absorption of SW radiation (with low entropy) and a further release of heat through respiration. In particular, $\eta_{\text {bio }}$ is more prominent in site \#19 located in the tropics where the photosynthetic rate is significant.

\subsubsection{Dynamics of $\sigma_{\mathrm{cz}, \mathrm{int}}$ Over Time}

Figure 9 shows five different sites where there is available information to compute $\mathrm{E}_{\mathrm{cz}, \text { in }}$ and $\eta_{\mathrm{cz}, \text { int }}$ at different stages of ecosystem development. Figure 9a,b,c and d shows $\mathrm{E}_{\mathrm{cz}, \mathrm{in}}, \mathrm{dE}_{\mathrm{cz}}, \mathrm{dE}_{\mathrm{cz}}$, and $\eta_{\mathrm{cz}, \text { int }}$, respectively across different stages of ecosystem development. The dynamics of $\eta_{\mathrm{cz} \text {,int }}$ are controlled by a trade off between $\eta_{\text {bio }}$ and $\eta_{\mathrm{Q}}$. In the following we further analyze these two components independently.

\section{Dynamics of $\eta_{\text {bio }}$}

Analysis of the data recorded in site \#20 (Figure 9 I.) shows that $\eta_{\text {bio }}$ is higher in the more developed stages. However in sites \#23 (Figure 9 II.), \#6 (Figure 9 IV.), and \#24 (Figure 9 V.) $\eta_{\text {bio }}$ tends to be lower in the more developed stages. In site \#29 (Figure 9 III.) the only site where more than two points with reliable information is available, we observe that 
$\eta_{\text {bio }}$ fluctuates across different stages following the dynamics observed in the respiration fluxes.

Note that $\eta_{\text {bio }}$ relies in the transformation of biochemical energy stored as reduced carbon (with low entropy) into heat (with higher entropy) (see Equation 9). This process is induced by oxidation of stored biochemical energy through respiration. As a result, $\eta_{\text {bio }}$ is strongly regulated by respiration fluxes. In Figure 9 we observe that in the more mature ecosystems the storage of energy $\mathrm{dE}_{\mathrm{cz}, \mathrm{pho}}$ increases. This storage refers to photosynthetic compounds of reduced carbon with low entropy (high exergy), which suggests that exergy tends to increase in the more developed stages. However, increase of $\mathrm{dE}_{\mathrm{cz}, \mathrm{pho}}$ is associated with lower respiration fluxes that in turn hinders $\eta_{\text {bio. }}$. In the end, we observe that in the more developed stages there are higher photosynthetic fluxes $\left(\mathrm{E}_{\mathrm{SW}, \mathrm{pho}}\right)$ but there is a preferential trend to increase the storage instead of respiring the entire input biomass, and as a result there is no clear pattern for $\eta_{\text {bio }}$.

Because $\eta_{\text {bio }}$ relies on respiration, $\eta_{\text {bio }}$ could be higher in stages with lower photosynthetic rates if the fluxes of respiration are significant. This pattern is observed in site \#6 (Figure 9 II.b and d), and site \#24 (Figure 9 V.b and d.) where $\eta_{\text {bio }}$ is higher in the less developed stages and the fluxes of photosynthesis are lower, but the fluxes of respiration are higher and are maintained at the expense of exergy that had been stored previously.

From the information analyzed in Figure 9 we observe three consistent patterns across the different stages of ecosystem development:

- $\mathrm{E}_{\mathrm{SW}, \mathrm{pho}}$ tends to increase in the more developed ecosystems, with higher photosynthetic fluxes.

- $\mathrm{dE}_{\mathrm{cz}, \mathrm{pho}}$ tends to increase in the more developed ecosystems, with higher exergy stored.

- There is no clear pattern for $\eta_{\text {bio }}$ with the development stage.

\section{Dynamics of $\eta_{Q}$}

Figure $9 \mathrm{~d}$ shows $\eta_{\mathrm{Q}}$ at five locations where it is possible to compare $\eta_{\mathrm{Q}}$ during different stages of ecosystem development. In order to provide a fair comparisson, $\eta_{\mathrm{Q}}$ reported in Figure 9d corresponds to computations performed over a common period of time with available records to estimate $\eta_{\mathrm{Q}}$ at all the different stages of development that are compared within a site. 
We observe that $\eta_{\mathrm{Q}}$ tends to decrease in the more developed stages as in sites \#6, \#20, \#23, and \#29. In site \#24 we do not observe significant differences in $\eta_{\mathrm{Q}}$ between the two stages that are compared. As mentioned above $\eta_{\mathrm{Q}}$ is function of the net heat flux into $\mathrm{CZ}$ and the gradients in temperature at which the incoming and outgoing heat fluxes occur. In turn, these variables are regulated by the released of $\mathrm{LE}$ and $\mathrm{H}$ fluxes from the surface. Higher $\mathrm{LE}$ and $\mathrm{H}$ fluxes enhance the dissipation of heat from the $\mathrm{CZ}$ and therefore they hinder the heat flux that is absorbed by the CZ. We observe that the variation of $\eta_{\mathrm{Q}}$ was inversely proportional to the emission of LE and $\mathrm{H}$ fluxes.

\section{Conclusions}

In this study we have recognized different formulations for the production of entropy that have been conceptualized in previous studies and are associated with different scales and processes in the Earth's Critical Zone $(\mathrm{CZ})$ :

- $\eta_{\mathrm{cz}}$ is the total production of entropy including all the exchange fluxes reaching and leaving the $\mathrm{CZ}$, and is regulated by the transformation of shorttwave (SW) radiation to other forms of energy. Around 97\% of $\eta_{\mathrm{cz}}$ is due to an instantaneous transformation of SW at the surface $\left(\eta_{\mathrm{cz}, \mathrm{rad}}\right)$, and as a result $\eta_{\mathrm{cz}}$ is enhanced by the amount of incoming SW radiation and is mainly controlled by the albedo of the land surface.

- $\eta_{\text {bio }}$ refers to the production of entropy due to biological processes, and is associated with the absorption of shortwave (SW) radiation in chemical compounds and its further dissipation in the form of heat after respiration. $\eta_{\text {bio }}$ is regulated in most part by respiration because it is the process that triggers the conversion of chemical energy with low entropy to heat with higher entropy. We observe that higher photosynthetic fluxes do not necessarily imply higher $\eta_{\text {bio }}$ if there is significant storage of photosynthetic products that hinders respiration. In addition, as pointed in Volk and Pauluis (2010) the highest $\eta_{\text {bio }}$ will occur during a disturbance such as a fire where the energy stored in chemical compounds will be transformed to heat in a short period of time.

- $\eta_{\mathrm{Q}}$ refers to the production of entropy due to fluxes of heat, and is related to incoming and outgoing heat fluxes that occur at different temperatures. Therefore, $\eta_{\mathrm{Q}}$ is regulated by the magnitude of the heat fluxes and the gradients in temperature that occur during these fluxes. 
- $\eta_{\mathrm{cz}, \text { int }}$ refers to the production of entropy due to internal processes by considering only the energy that is absorbed, $\eta_{\mathrm{cz}, \text { int }}=\eta_{\mathrm{bio}}+\eta_{\mathrm{Q}}$.

We observed different patterns in the entropy production computed from these formulations. Although all these formulations refer to entropy production they include different processes and refer to different scales of dynamics. Therefore, the conclusions or hypotheses that are extracted from these formulations should be considered and analyzed independently.

In this study we have made an attempt to quantify the production of entropy in the $\mathrm{CZ}$ with information recorded in FLUXNET towers. We have taken advantage of the data recorded in the Ameriflux Network to quantify the production of entropy in different climates and at different stages of ecosystem development. Based on the results obtained in this study we did not find a clear evidence that supports a maximization of entropy production in more vegetated areas or an increment across the different stages of development, for any of the entropy production formulations that were considered:

(i) We computed the productions of entropy in different ecosystems and climates. On the one hand, when the production of entropy including all the energy fluxes $\left(\eta_{\mathrm{cz}}\right)$ was compared we observed that different ecosystems with rather different dynamics of energy dissipation could attain similar $\eta_{\mathrm{cz}}$. This result is similar to previous findings reported by Volk and Pauluis (2010) where rather different atmospheric conditions could lead to the same entropy production. In fact, we observed that $\eta_{\mathrm{cz}}$ was highest in the arid and semi arid climates. This is contrary to intuition and the reason is because these ecosystems receive higher incoming SW radiation (less clouds) and dissipate the energy in the form of LW instead of heat (sensible or latent) that enhance the production of entropy. This in turn suggests that dry ecosystems with little vegetation could have a significant production of entropy because they are receiving more SW radiation, and using LW (less latent heat) as a main alternative to dissipate the incoming fluxes of energy. These results disclaim previous studies suggesting that vegetation is a major evidence for the Maximum Entropy Production Principle for macroscopic systems (ModMEPP) because they enhance the production of entropy. On the other hand, when the production of entropy including only the energy fluxes absorbed within the $\mathrm{CZ}$ were considered we observed that there is a production of entropy from heat $\left(\eta_{\mathrm{Q}}\right)$ and photosynthetic $\left(\eta_{\text {bio }}\right)$ fluxes at all the sites, but their magnitudes vary according to the type of ecosystem and climate. For instance, in 
site \#19 (br-sa) located in the Amazon $\eta_{\text {bio }}$ was dominant, while in dessert ecosystems such as in site \#24 (us-se) $\eta_{\mathrm{Q}}$ was dominant. These patterns reflect only the presence of vegetation. As expected $\eta_{\text {bio }}$ will be higher in systems where photosynthetic fluxes are more prominent. Similarly, in the absence of vegetation (lower latent heat) there is a higher heat absorbed by the system that in turn enhances $\eta_{\mathrm{Q}}$. However, there are no clear patterns for the total internal entropy production $\left(\sigma_{\mathrm{cz}, \text { int }}=\sigma_{\mathrm{bio}}+\sigma_{\mathrm{Q}}\right)$ as different ecosystems with rather different configurations could attain similar values of $\sigma_{\mathrm{cz}, \text { int }}$.

(ii)We also analyzed the production of entropy across different stages of ecosystem development. In a previous study Holdaway et al. (2010) compared $\eta_{\mathrm{cz}}$ across different stages of development in the Amazon and found that $\eta_{\mathrm{cz}}$ tends to increase in the more developed stages. They claimed these dynamics support ModMEPP because the ecosystems were converging towards a higher production of entropy. Here, we analyzed $\eta_{\mathrm{cz}}$ across different stages of development in nine sites exposed to different climates. We observed the surface albedo tends to reduce in the most developed stages and therefore $\eta_{\mathrm{cz}}$ tends to increase in the more developed stages as suggested by Holdaway et al. (2010). However, we found these dynamics do not hold in three of the nine sites analyzed where the albedo did not increase in the more developed stages. This suggests that $\eta_{\mathrm{cz}}$ do not always increase in the more developed stages as claimed by Holdaway et al. (2010). The patterns for $\sigma_{\text {bio }}$ and $\sigma_{\mathrm{Q}}$ across different stages of development are even less clear. On the one hand, $\sigma_{\text {bio }}$ varies in time following dynamics in respiration and photosynthesis. We observed that more developed ecosystems tend to increase the photosynthetic flux but also they increase the storage of photosynthetic products. This pattern supports the maximum exergy storage production principle (Mejer and Jorgensen, 1979). However, increase in storage comes with the cost of hindering respiration that in the end results in no clear pattern for $\sigma_{\text {bio }}$, which can increase or decrease during the different stages of development. These dynamics suggest that ModMEPP in the light of $\sigma_{\text {bio }}$ is not valid for ecosystem development. On the other hand, we observed that $\sigma_{\mathrm{Q}}$ tends to reduce in the more developed stages because less amount of heat is going into the $\mathrm{CZ}$, which is opposite to ModMEPP.

Acknowledgments The data for this paper are available at the Ameriflux data system. We acknowledge all the Ameriflux sites and their principal investigators for their data records. Funding for Ameriflux data resources was provided by the U.S. Department of Energy's Office of Science. 


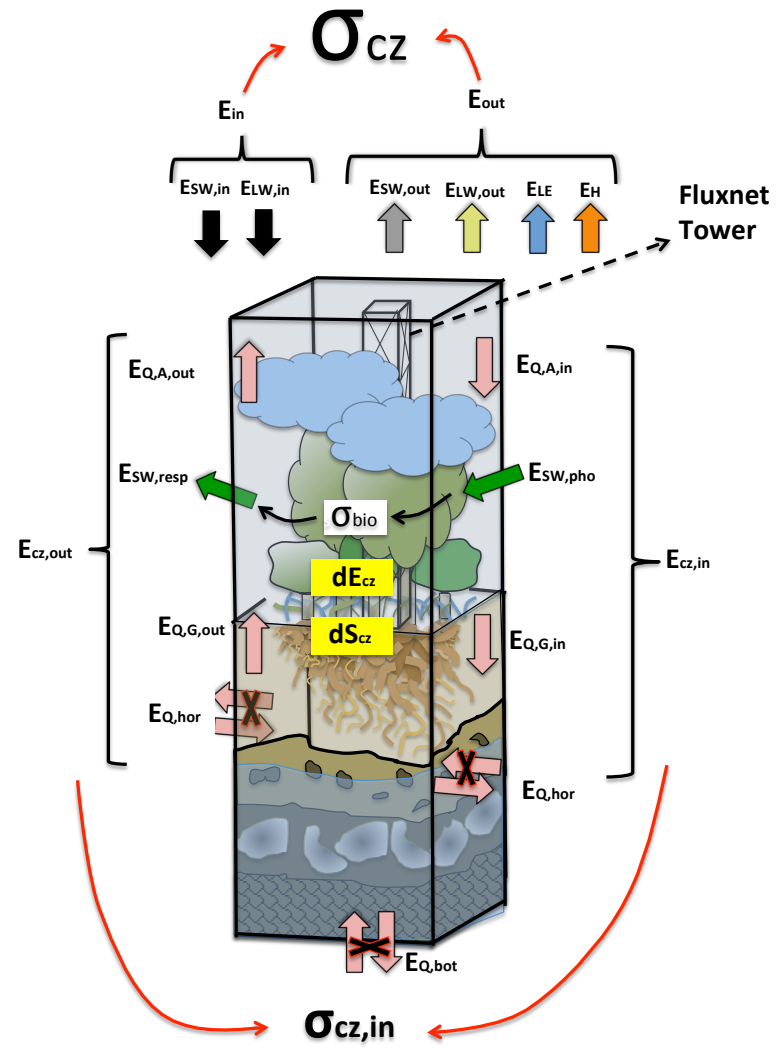

The Critical Zone
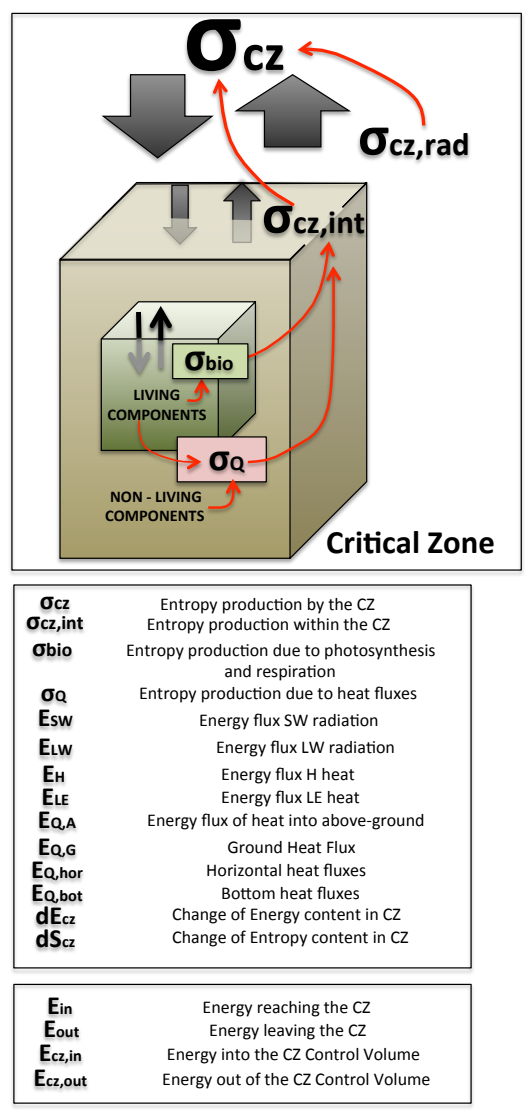

Figure 1: Schematic of the Critical Zone (CZ) showing different entropy productions that are examined in this study. The control volume of the $\mathrm{CZ}$ is considered as an imaginary boundary delineated by the footprint of the FLUXNET tower. Horizontal and bottom transfers of heat (i.e., $\mathrm{E}_{\mathrm{Q} \text {,hor }}$ and $\mathrm{E}_{\mathrm{Q} \text {,bot }}$ ) are neglected. The $\mathrm{CZ}$ receives an important source of energy $\left(E_{\text {in }}\right)$ in the form of shortwave $(\mathrm{SW})$ and longwave radiation $(\mathrm{LW})$. A significant fraction of this energy is dissipated instantaneously in different forms such as SW and LW radiation, latent heat (LE), and sensible heat (H). Transformation of SW radiation to heat accounts for an instantaneous production of entropy at the top of the $\mathrm{CZ}$ $\left(\sigma_{c z}\right)$. A small fraction of the incoming $\mathrm{E}_{\text {in }}$ is absorbed by the $\mathrm{CZ}$ in the form of heat and chemical compounds through photosynthesis $\left(\mathrm{E}_{\mathrm{cz}, \mathrm{in}}\right)$. The dissipation of $\mathrm{E}_{\mathrm{cz}, \text { in }}$ in the form of heat results in a production of entropy that is directly related to internal processes within the $\mathrm{CZ}\left(\sigma_{\mathrm{cz}, \text { int }}\right)$. On one hand, the absorption of $\mathrm{SW}$ radiation through photosynthesis and further release as heat through respiration produces the most significant entropy production in living components $\left(\sigma_{\text {bio }}\right)$. On the other hand, the absorption and dissipation of heat fluxes within the $\mathrm{CZ}$ also produce entropy and is denoted as $\left(\sigma_{\mathrm{Q}}\right)$. The inlet figure shows a schematic representation of $\sigma_{\mathrm{cz}}, \sigma_{\mathrm{cz}, \text { int }}, \sigma_{\mathrm{bio}}$, and $\sigma_{\mathrm{Q}}$ 


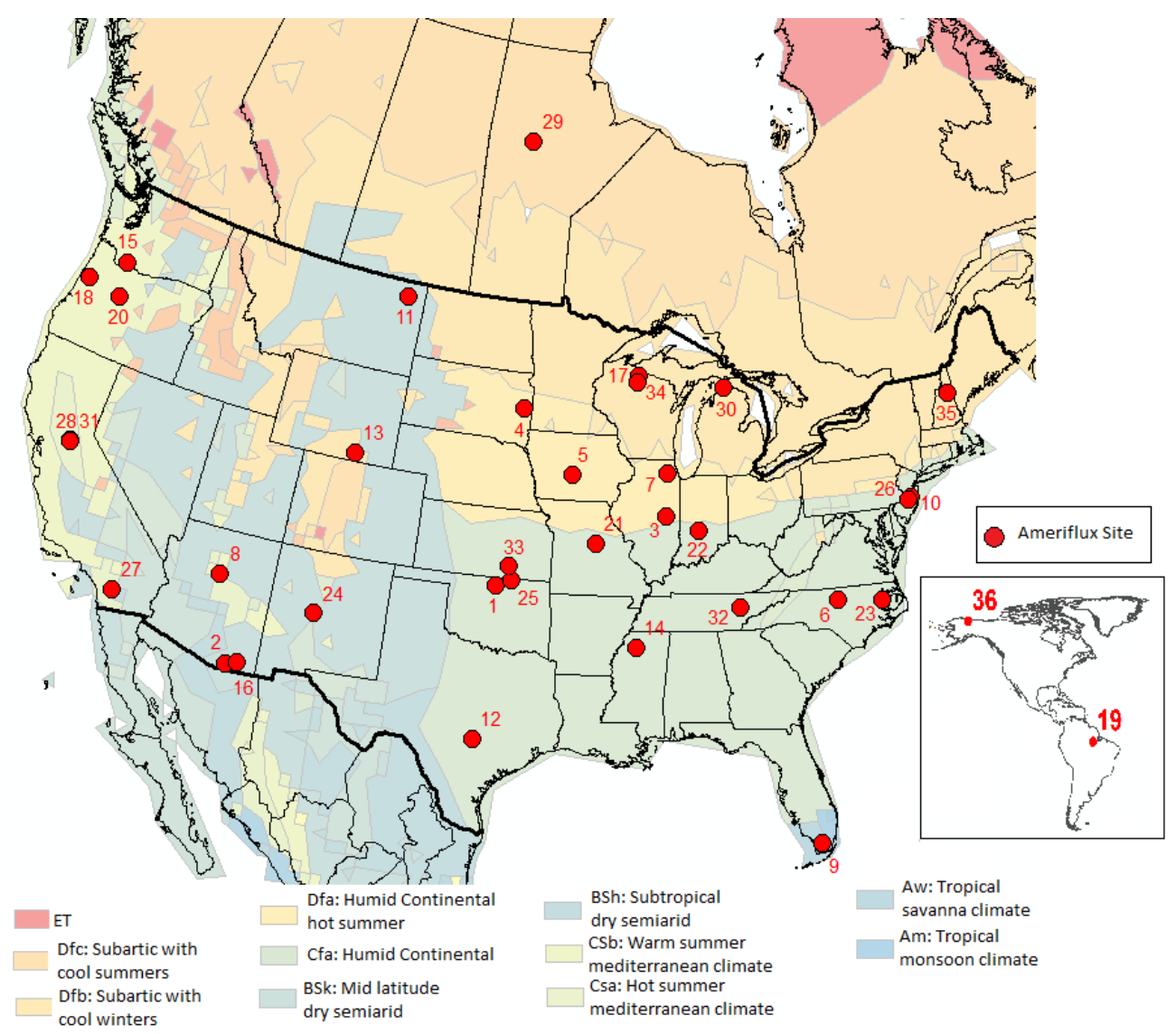

Figure 2: Map of the Ameriflux sites selected for this study. The background shows the Kö-Geiger climate classification according to Kottek et al. (2006). 

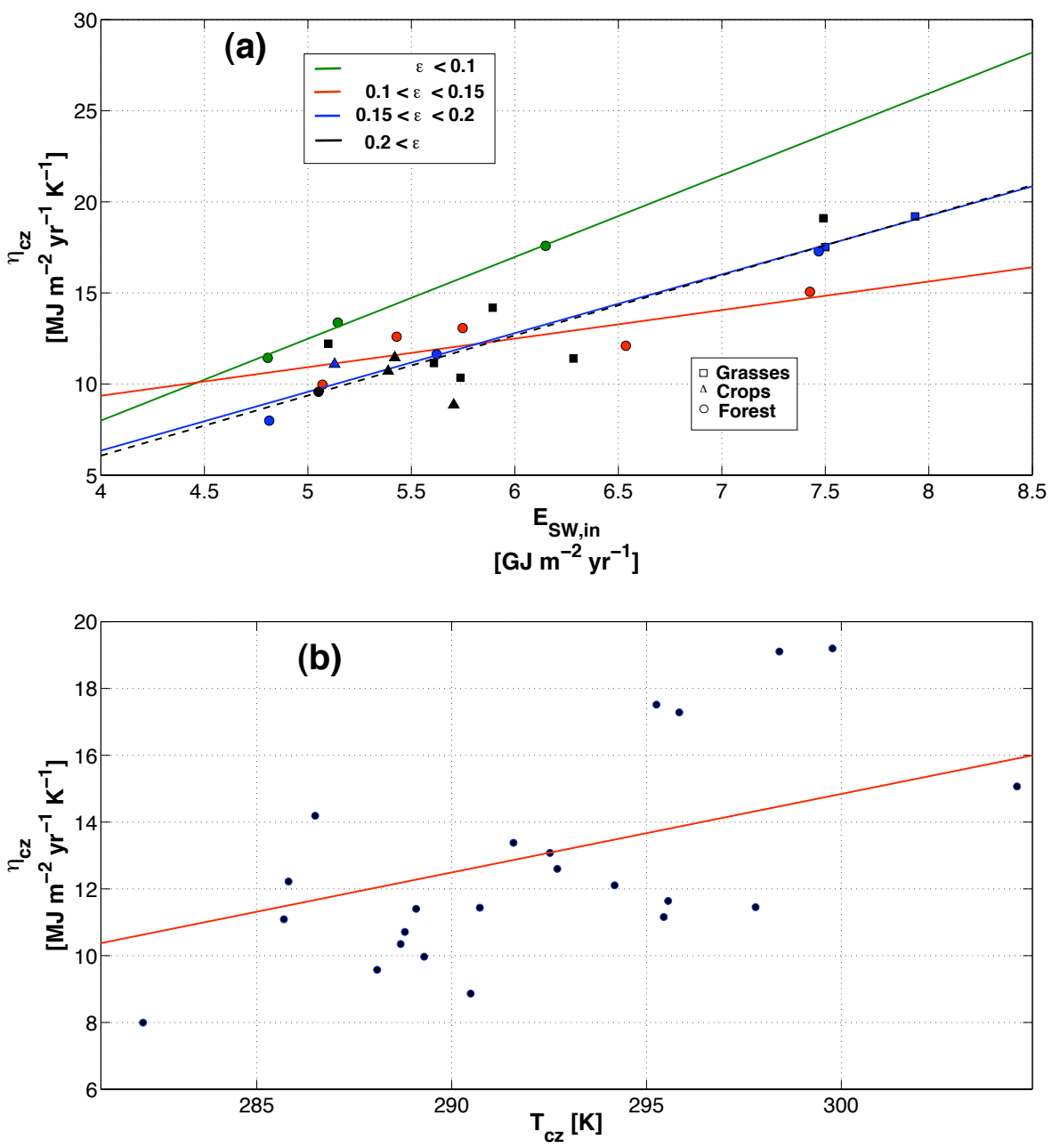

Figure 3: Top panel shows (a) the relationship between the total production of entropy by the Critical Zone $(\mathrm{CZ})$ in a year $\left(\eta_{\mathrm{cz}}\right)$ and the incoming $\mathrm{SW}$ radiation $\left(\mathrm{E}_{\mathrm{SW} \text {,in }}\right)$ for different sites with available information to compute $\eta_{\mathrm{cz}}$. In this figure we differentiate between grasses (squares), crops (triangles), and forrest (circles). In addition, we grouped the sites based on the albedo and perform the fitting for these groups separately. Bottom panel shows (b) the relationship between $\eta_{\mathrm{cz}}$ and the effective temperature of the $\mathrm{CZ}\left(\mathrm{T}_{\mathrm{cZ}}\right)$. 

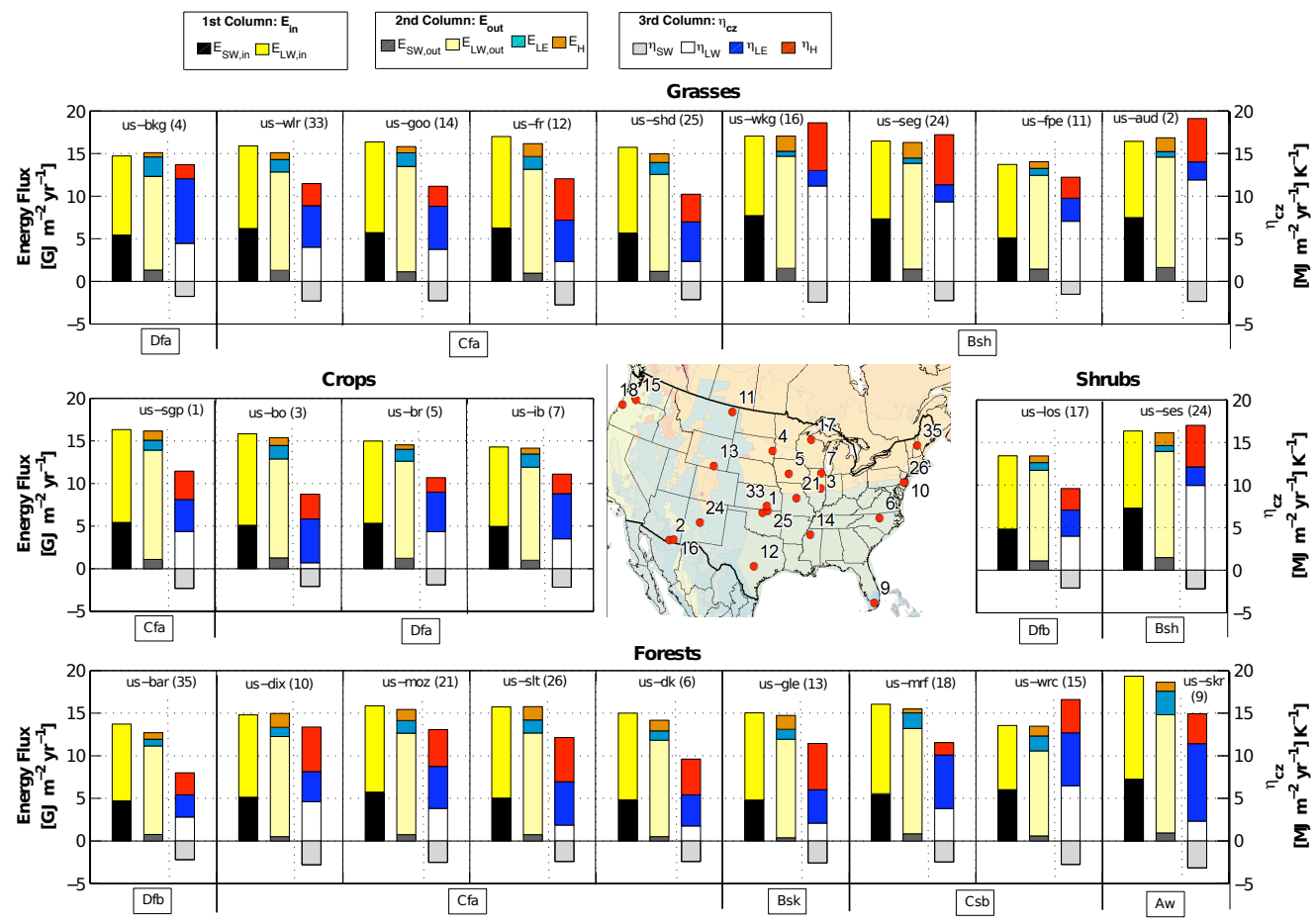

Figure 4: Energy balance (first two bars) and total production of entropy in the Critical Zone in a year $\left(\eta_{\mathrm{cz}}\right)$ (3rd bar) based on records from selected FLUXNET towers in different locations. The numbers above the bars represent the Ameriflux tower as displayed in Figure 2 and listed in Table 1. The code below bars represents the climatic classification as displayed in Figure 2. 

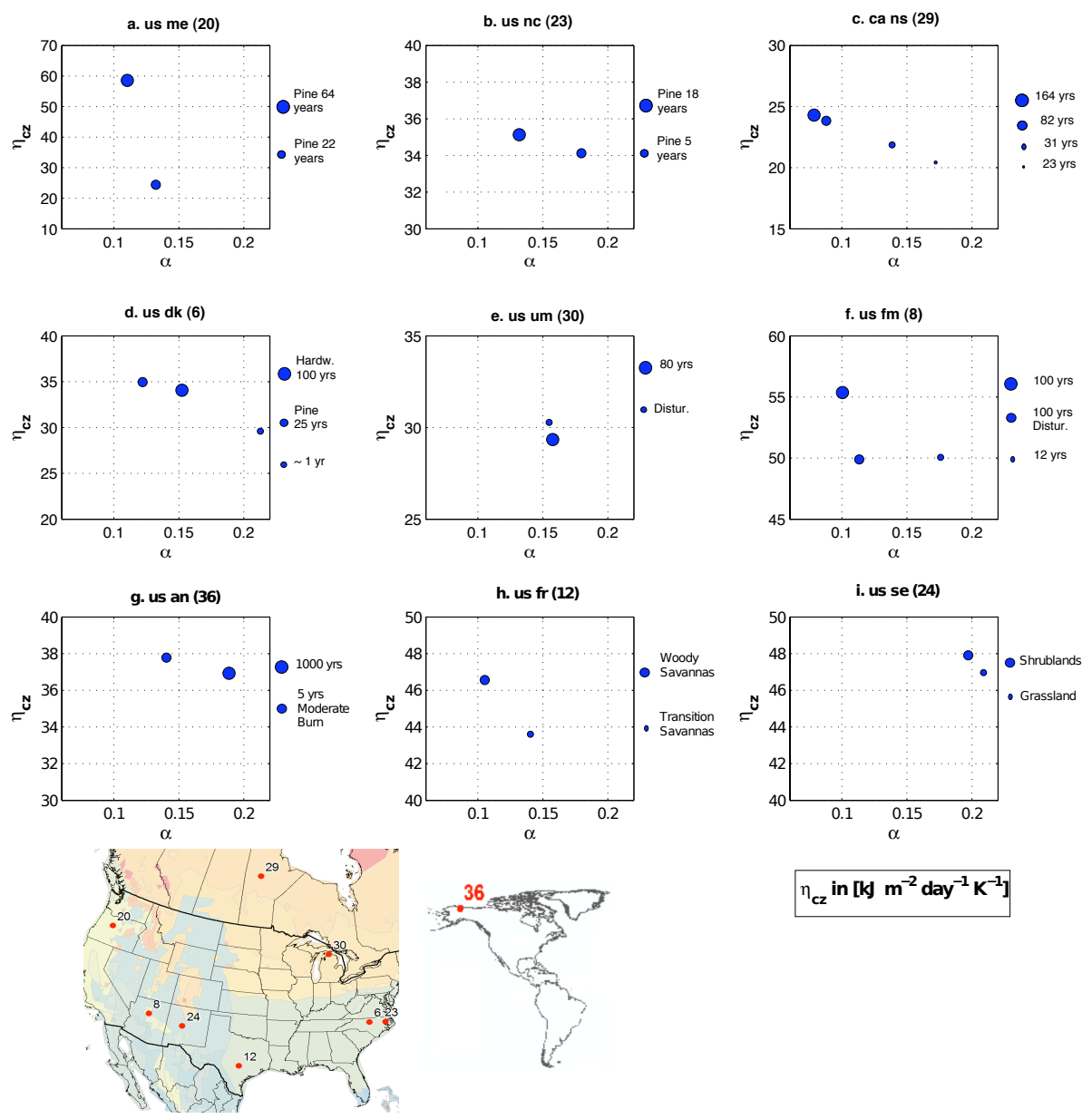

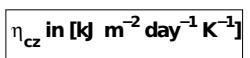

Figure 5: Patterns of $\eta_{\mathrm{cz}}$ across different stages of ecosystem development for nine sites where information at all the different stages of development is available. In most cases these records were not available for an entire year. Thus entropy production is presented per day instead of year, and represents the averaged daily entropy generated during the period with available data. Data in this figure is presented in Section 2 in the Online Supplement. 


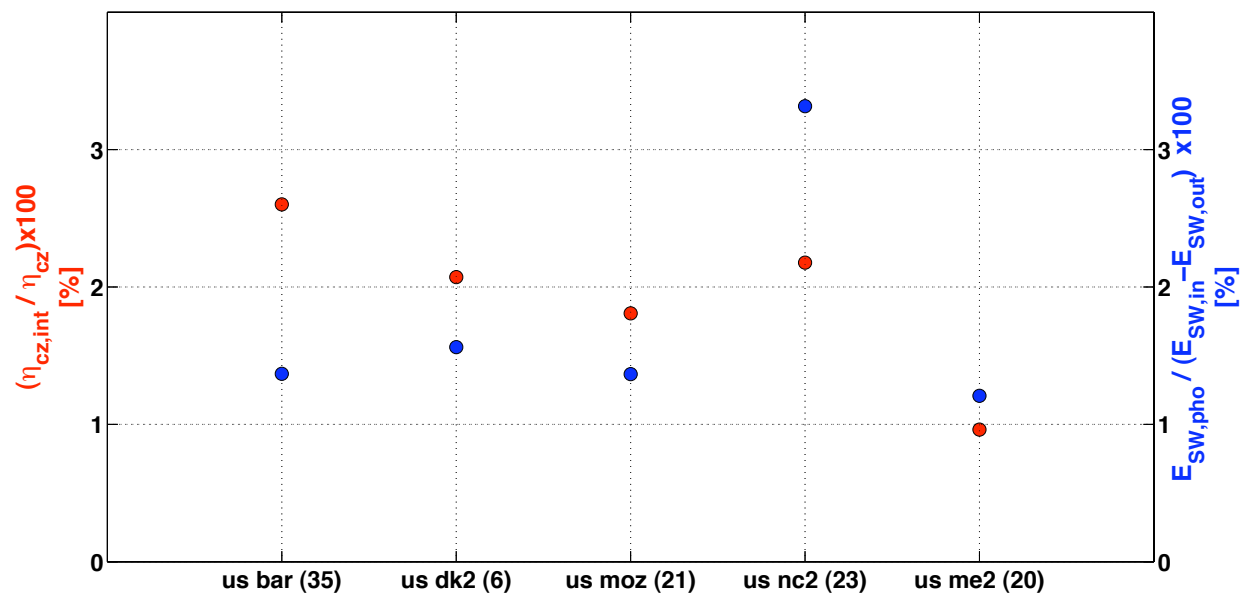

Figure 6: (Left) Ratio between the internal $\left(\eta_{\mathrm{cz}, \text { int }}\right)$ and total $\left(\eta_{\mathrm{cz}}\right)$ entropy production over a year time period (red dots), and (Right) ratio between the photosynthetic flux $\left(\mathrm{E}_{\mathrm{SW}, \mathrm{pho}}\right)$ and the energy flux of incoming shortwave radiation that is transformed to other forms of energy (including longwave radiation, photosynthesis, and heat, blue dots). This figure includes only sites where it is possible to compute both $\sigma_{\mathrm{cz}}$ and $\sigma_{\mathrm{cz} \text {,int }}$ for at least one year. See Table 1 and Figure 2 for the specifics of the locations indicated. 

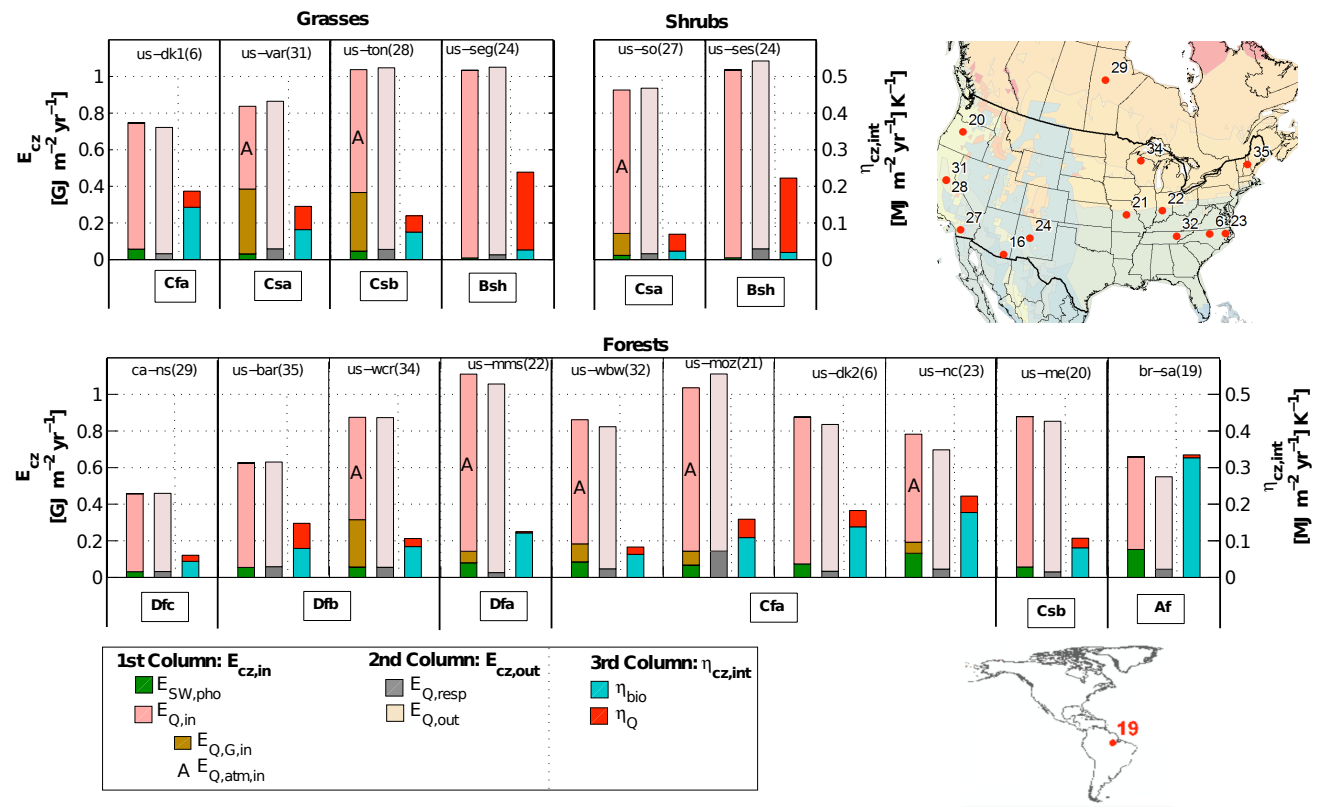

Figure 7: Energy balance (first two bars) and the production of entropy within the Critical Zone $\left(\sigma_{\mathrm{cz}, \text { int }}\right)$ (3rd bar), including only the fluxes of energy that are absorbed by the CZ. The numbers above the bars represent the Ameriflux tower as displayed in Figure 2 and listed in Table 1. The code below the bars represents the climatic classification as displayed in Figure 2. 


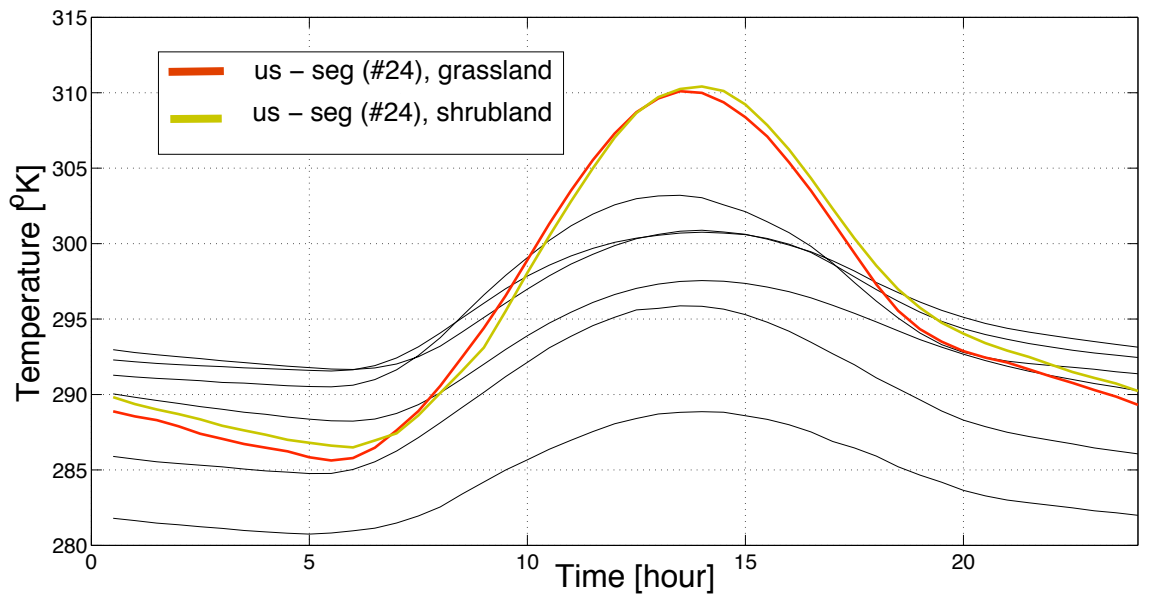

Figure 8: Mean annual diurnal temperature at the Critical Zone surface $\left(\mathrm{T}_{\mathrm{cz}}\right)$ computed with Boltzmann Equation from outgoing longwave (LW) radiation records (Equation 14). This figure shows the diurnal temperature for the sites displayed in Figure 7. However, only those sites with available information of outgoing LW radiation are displayed. In particular, we highlight the diurnal patterns in site \#24 (us-se) where the amplitude in the mean diurnal temperature is significantly higher when compared with all the other sites. Higher amplitude in surface temperature in site \#24 allows a higher production of entropy in the form of heat $\left(\eta_{\mathrm{q}}\right)$ as shown in Figure 7. 

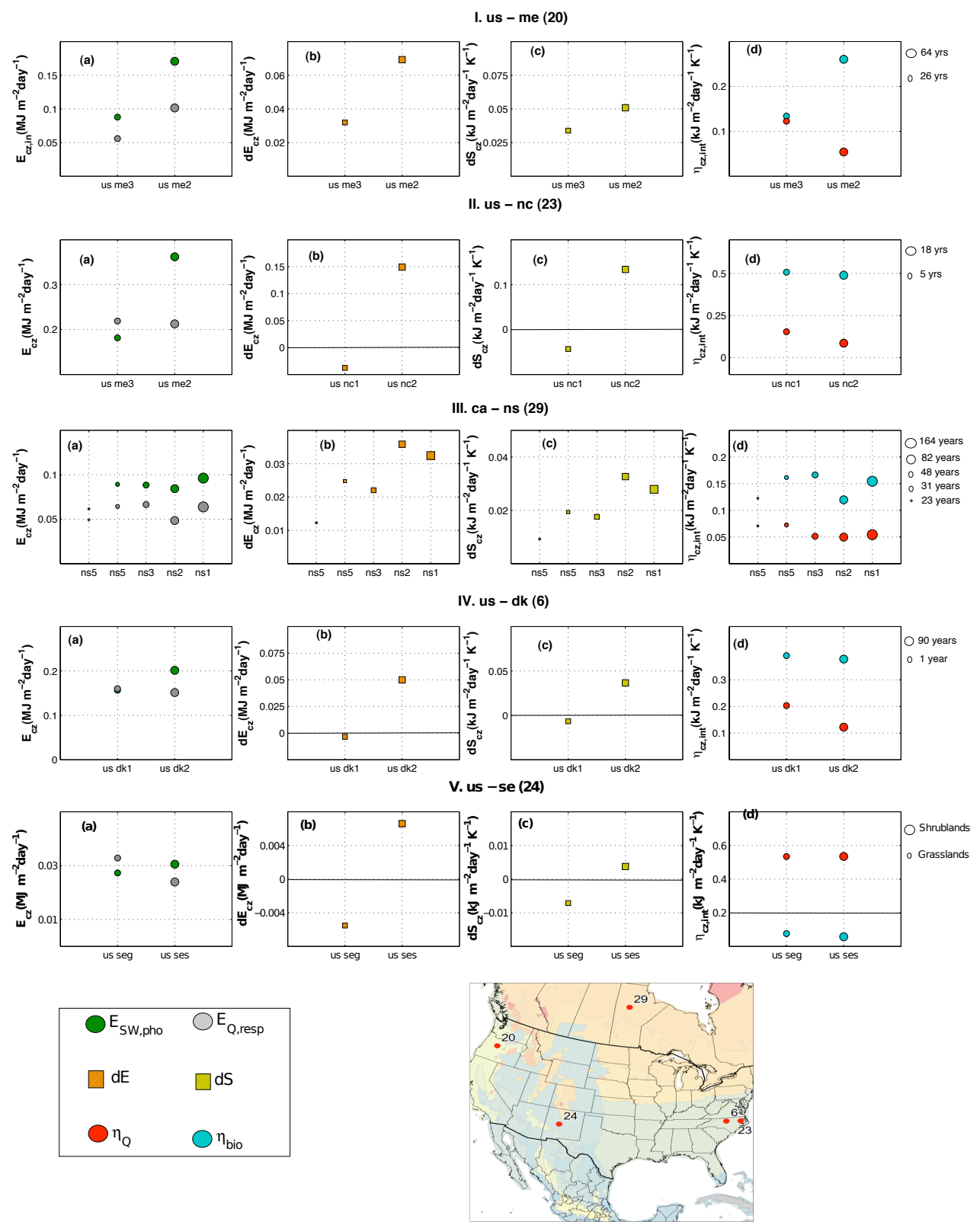

Figure 9: Patterns of (a) incoming energy absorbed by the Critical Zone (CZ), (b) rate of change of energy within the $\mathrm{CZ}\left(\mathrm{dE}_{\mathrm{cz}}\right),(\mathbf{c})$ rate of change of entropy within the $\mathrm{CZ}$ $\left(\mathrm{dS}_{\mathrm{cz}}\right)$, and (d) internal entropy production within the $\mathrm{CZ}\left(\eta_{\mathrm{cz}, \text { int }}\right)$. There are five different rows - with one row for each independent site- showing the respective dynamics where information at all the different stages of ecosystem development is available. In most cases these records were not available for an entire year. Thus entropy production is presented per day instead of year, and represents the averaged daily entropy generated during the period with available data. 
Table 1: List of Ameriflux sites used in this study. See Figure 3 for the specific locations of these sites and the legends of climatic regions.

\begin{tabular}{|c|c|c|c|c|c|c|c|c|c|}
\hline Number & Code & Location & Climate & Used as * & Number & Code & Location & Climate & Used as * \\
\hline 1. & us-sgp & US-OK & $\mathrm{Cfa}$ & 1 & 2. & us-aud & US-AZ & Bsh & 1 \\
\hline 3. & us-bo & US-IL & Dfa & 1 & 4. & us-bkg & US- SD & Dfa & 1 \\
\hline 5. & us-br & US-IA & Dfa & 1 & 6. & us-dk & US- NC & $\mathrm{Cfa}$ & $1,2 \mathrm{a}, \mathrm{b}$ \\
\hline 7. & us-ib & US-IL & Dfa & 1 & 8. & us-fm & US- AZ & Bsh & a \\
\hline 9. & us-skr & US-FL & Aw & 1 & 10. & us-dix & US-NJ & $\mathrm{Cfa}$ & 1 \\
\hline 11. & us-fpe & US-MT & Bsh & 1 & 12. & us-fr & US-TX & $\mathrm{Cfa}$ & $1 \mathrm{a}$ \\
\hline 13. & us-gle & US-WY & Bsk & 1 & 14. & us-goo & US-MS & $\mathrm{Cfa}$ & 1 \\
\hline 15. & us-wrc & US-WA & $\mathrm{Dfb}$ & 1 & 16. & us-wkg & US-AZ & Bsh & 1 \\
\hline 17. & us-los & US-WI & $\mathrm{Dfb}$ & 1 & 18. & us-mrf & US-OR & $\mathrm{Csb}$ & 1 \\
\hline 19. & br-sa & BR-PA & Aw & 2 & 20. & us-me & US-OR & Csb & $2 \mathrm{a}, \mathrm{b}$ \\
\hline 21. & us-moz & US-MO & $\mathrm{Cfa}$ & 1,2 & 22. & us-mms & US-IN & Dfa & 2 \\
\hline 23. & us-nc & US-NC & Cfa & $2 \mathrm{a}, \mathrm{b}$ & 24. & us-se & US-AZ & $\mathrm{Bsh}$ & $1,2 \mathrm{a}, \mathrm{b}$ \\
\hline 25. & us-shd & US-OK & $\mathrm{Cfa}$ & 1 & 26. & us-slt & US-NJ & $\mathrm{Cfa}$ & 1 \\
\hline 27. & us-so & US-CA & Csa & 2 & 28. & us-ton & US-CA & $\mathrm{Csb}$ & 2 \\
\hline 29. & ca-ns & CA-MB & $\mathrm{Dfc}$ & $2 \mathrm{a}, \mathrm{b}$ & 30. & us-umb & US-MI & $\mathrm{Dfb}$ & $\mathrm{a}$ \\
\hline 31. & us-var & US-CA & Csa & 2 & 32. & us-wbw & US-TN & $\mathrm{Cfa}$ & 2 \\
\hline 33. & us-wlr & US-KS & $\mathrm{Cfa}$ & 1 & 34. & us-wcr & US-WI & Dfb & 2 \\
\hline 35. & us-bar & US-NH & $\mathrm{Dfb}$ & 1,2 & 36. & us-an & US-AK & Dfc & $\mathrm{a}$ \\
\hline $\begin{array}{l}*^{*} 1 . \text { Used } \\
{ }^{*} 2 \text {. Used } \\
*^{*} \text { a. Used } \\
{ }^{*} \text { b. Used }\end{array}$ & $\begin{array}{l}\text { compu } \\
\text { compu } \\
\text { compu }\end{array}$ & $\begin{array}{l}\eta_{\mathrm{cz}} \\
\eta_{\mathrm{cz}, \text { int }} \\
\eta_{\mathrm{cz}} \text { for } \\
\eta_{\mathrm{cz}, \text { int }}\end{array}$ & st ty & $\begin{array}{l}\text { develo } \\
\text { of dev }\end{array}$ & $\mathrm{nt}$ & & & & \\
\hline
\end{tabular}


Table 2: List of parameters used for the computation of entropy.

\begin{tabular}{cccc}
\hline \hline Property & Symbol & Units & Magnitude \\
\hline \hline Sun Temperature & $\mathrm{T}_{\text {sun }}$ & $\mathrm{K}$ & 5760 \\
Stefan-Boltzmann Constant & $\lambda$ & $\mathrm{Wm}^{-2} \mathrm{~K}^{-4}$ & $5.67 \times 10^{-8}$ \\
Specific Biomass Enthalpy & $h_{\mathrm{BIO}}$ & ${\mathrm{J} \mu \mathrm{mol}^{-1}}^{1} 0.506$ \\
Critical Zone Emissivity & $\varepsilon$ & - & ${ }^{2} 0.95$ \\
& \multicolumn{3}{c}{} \\
\hline${ }^{1}$ Nikolov (1995); ${ }^{2}$ Holdaway et al. (2010), Campbell and Norman (1998) \\
\hline \hline
\end{tabular}


Aoki, I., Entropy budgets of deciduous plant leaves and theorem of oscillating entropy production, F Mathematical Biology, 49(4), 449-460, 1987.

Aoki, I., Entropy budget of conifer branches, Botanical Magazine-Tokio, 102(1065), 133-141, 1989.

Aoki, I., Entropy production in living systems: from organisms to ecosystems, Thermochimica Acta, 250(2), 359-370, doi:10.1016/0040-6031(94)02143C, 1995 .

Bejan, A., Entropy generation minimization, exergy analysis, and the constructal law, Arabian Journal for Science and Engineering, 38(2), 329-340, doi:10.1007/s13369-012-0444-6, 2013.

Bendoricchio, G., and S. E. Jorgensen, Exergy as goal function of ecosystems dynamic, Ecological Modelling, 102(1), 5-15, doi:10.1016/S03043800(97)00091-4, 1997.

Campbell, G., and D. Norman, An Introduction to Environmental Biophysics, Springer, 1998.

Danielewicz-Ferchmin, I., and A. R. Ferchmin, A check of prigogine's theorem of minimum entropy production in a rod in a nonequilibrium stationary state, American Journal of Physics, 68(10), 962-965, doi: 10.1119/1.1286046, 2000.

Fisher, J. B., D. D. Baldocchi, L. Misson, T. E. Dawson, and A. H. Goldstein, What the towers don't see at night: nocturnal sap flow in trees and shrubs at two ameriflux sites in california, Tree Physiology, 27(4), 597-610, 2007.

Holdaway, R. J., A. D. Sparrow, and D. a. Coomes, Trends in entropy production during ecosystem development in the Amazon Basin., Philosophical transactions of the Royal Society of London. Series B, Biological sciences, 365(1545), 1437-47, doi:10.1098/rstb.2009.0298, 2010.

Hoover, W. G., Note on "comment on 'a check of prigogine's theorem of minimum entropy production in a rod in a nonequilibrium stationary state,' by irena danielewicz-ferchmin and a. ryszard ferchmin" [am. j. phys. 68 (10), 962-965 (2000)], by peter palffy-muhoray [am. j. phys. 69 (7), 825-826 (2001)], American Journal of Physics, 70 (4), 452-454, doi: 10.1119/1.1432974, 2002. 
Ichiro, A., Entropy productions on the earth and other planets of the solar system, Journal of the Physical Society of Japan, 52(3), 1075-1078, doi: 10.1143/JPSJ.52.1075, 1983-03-15.

Jaynes, E. T., The minimum entropy production principle, Annual Review of Physical Chemistry, 31(1), 579-601, 1980.

Jørgensen, S., B. Fath, S. Bastianoni, J. Marques, F. Muller, S. Nielsen, B. Patten, E. Tiezzi, and R. . Ulanowicz, A New Ecology: Systems Perspective, Elsevier Science, 2011.

Kay, J., Handbook of Ecosystem Theories and Management, CRC Press, chap. Ecosystems as self-organizing holarchic open systems: Narratives and the Second Law of Thermodynamics, pp. 135-160, Lewis Publishers, San Diego, doi:10.1016/B978-0-12-386941-8.00008-3, 2000.

Kleidon, A., Beyond gaia: Thermodynamics of life and earth system functioning, Climatic Change, 66(3), 271-319, 2004.

Kleidon, A., Y. Malhi, and P. M. Cox, Maximum entropy production in environmental and ecological systems., Philosophical transactions of the Royal Society of London. Series B, Biological sciences, 365(1545), 1297302, doi:10.1098/rstb.2010.0018, 2010.

Kleidon, A., E. Zehe, and H. Lin, Hydropedology, chap. Thermodynamic Limits of the Critical Zone and their Relevance to Hydropedology, pp. 243-281, Elsevier Academic Press INC, San Diego, doi:10.1016/B978-012-386941-8.00008-3, 2012.

Kondepudi, D., and I. . . Prigogine, Modern thermodynamics: from heat engines to dissipative structures, John Wiley, 1998.

Kottek, M., J. Grieser, C. Beck, B. Rudolf, and F. Rubel, World map of the köppen-geiger climate classification updated, Meteorologische Zeitschrift, 15(3), 259-263, doi:doi:10.1127/0941-2948/2006/0130, 2006.

Lorenz, R. D., Planets, life and the production of entropy, International Journal of Astrobiology, 1(1), 3-13, 2002.

Lucia, U., Stationary open systems: A brief review on contemporary theories on irreversibility, Physica A: Statistical Mechanics and its Applications, 
392(5), 1051-1062, doi:http://dx.doi.org/10.1016/j.physa.2012.11.027, 2013.

Lucia, U., Entropy generation and cell growth with comments for a thermodynamic anticancer approach, Physica A: Statistical Mechanics and its Applications, 406(0), 107-118, doi: http://dx.doi.org/10.1016/j.physa.2014.03.053, 2014.

Martyushev, L., Entropy and entropy production: Old misconceptions and new breakthroughs, Entropy, 15(4), 1152-1170, 2013.

Martyushev, L., and V. Seleznev, Maximum entropy production principle in physics, chemistry and biology, Physics Reports, 426(1), 1-45, doi: 10.1016/j.physrep.2005.12.001, 2006.

Mejer, H., and S. Jorgensen, Energy and ecological buffer capacity, in Proc. Conf. on Ecological Modelling, 28th August-2nd, September 1978, Copenhagen. International Society for Ecological Modelling, edited by J. S.E., 1979.

Meysman, F. J. R., S. Bruers, F. J. R. Meysman, and S. Bruers, Ecosystem functioning and maximum entropy production : a quantitative test of hypotheses Ecosystem functioning and maximum entropy production : a quantitative test of hypotheses, Philosophical transactions of the Royal Society of London. Series B, Biological sciences, doi:10.1098/rstb.2009.0300, 2010 .

National Research Council. Basic Research Opportunities in Earth Science, The National Academies Press., 2001.

Nikolov, N., Coupling biochemical and biophysical processes at the leaf level: an equilibrium photosynthesis model for leaves of C3 plants, Ecological Modelling, 80(2-3), 205-235, doi:10.1016/0304-3800(94)00072-P, 1995.

Oliphant, A. J., C. S. B. Grimmond, H. N. Zutter, H. P. Schmid, H. B. Su, S. L. Scott, B. Offerle, J. C. Randolph, and J. Ehman, Heat storage and energy balance fluxes for a temperate deciduous forest, Agricultural and Forest Meteorology, 126(3-4), 185-201, doi: http://dx.doi.org/10.1016/j.agrformet.2004.07.003, 2004. 
Ozawa, H., A. Ohmura, R. D. Lorenz, and T. Pujol, The second law of thermodynamics and the global climate system: A rewview of the maximum entropy production principle, Reviews of Geophysics, 41(4), doi: 10.1029/2002RG000113, 2003.

Palffy-Muhoray, P., Comment on "a check of prigogine's theorem of minimum entropy production in a rod in a nonequilibrium stationary state" by irena danielewicz-ferchmin and a. ryszard ferchmin [am. j. phys. 68 (10), 962-965 (2000)], American Journal of Physics, 69(7), 825-826, doi: 10.1119/1.1371916, 2001.

Paltridge, G. W., Global dynamics and climate - a system of minimum entropy exchange, Quarterly Journal of the Royal Meteorological Society, 101(429), 475-484, doi:10.1002/qj.49710142906, 1975.

Pauluis, O., and I. M. Held, Entropy budget of an atmosphere in radiativeconvective equilibrium. part i: Maximum work and frictional dissipation, Journal of the Atmospheric Sciences, 59(2), 125-139, 2002.

Porada, P., A. Kleidon, and S. J. Schymanski, Entropy production of soil hydrological processes and its maximisation, Earth Syst. Dynam., 2(2), 179-190, doi:10.5194/esd-2-179-2011, 2011.

Prigogine, I., Etude thermodynamique des phènomënes irrèversibles, Review, Université libre de Bruxelles, 1947.

Quijano, J., and H. Lin, Entropy in the Critical Zone: A Comprehensive Review, ENTROPY, 16(6), 3482-3536, doi:10.3390/e16063482, 2014.

Rasmussen, C., P. A. Troch, J. Chorover, P. Brooks, J. Pelletier, and T. E. Huxman, An open system framework for integrating critical zone structure and function, Biogeochemistry, 102(1-3), 15-29, doi:10.1007/s10533-0109476-8, 2011.

Schneider, E., and J. Kay, Life as a manifestation of the second law of thermodynamics, Mathematical and Computer Modelling, 19(6-8), 25 - 48, doi:10.1016/0895-7177(94)90188-0, 1994.

Ulanowicz, R. E., and B. M. Hannon, Life and the Production of Entropy, Proc. R. Soc. Lond., B 232, 181-192, doi:10.1098/rspb.1987.0067, 1987. 
Volk, T., and O. Pauluis, It is not the entropy you produce, rather, how you produce it, Philosophical transactions of the royal society B-Biological Sciences, 365(1545, SI), 1317-1322, doi:10.1098/rstb.2010.0019, 2010.

Westhoff, M. C., E. Zehe, and S. J. Schymanski, Importance of temporal variability for hydrological predictions based on the maximum entropy production principle, Geophysical Research Letters, 41(1), 67-73, doi:10.1002/2013GL058533, 2014.

Wilson, K., et al., Energy balance closure at fluxnet sites, Agricultural and Forest Meteorology, 113(1-4), 223-243, doi: http://dx.doi.org/10.1016/S0168-1923(02)00109-0, 2002.

Wright, S., D. Scott, J. Haddow, and M. Rosen, On the entropy of radiative heat transfer in engineering thermodynamics, International Journal of Engineering Science, 39(15), 1691 - 1706, doi:10.1016/S0020-7225(01)000246, 2001.

Wu, W., and Y. Liu, Radiation entropy flux and entropy production of the earth systems, Reviews of Geophysics, 48(2008RG000275), 1-27, doi: 10.1029/2008RG000275.1.INTRODUCTION, 2010.

Ziegler, H., Progress in Solid Mechanics, vol. 4,, pp. 243-281, I.N. Sneddon, R.Hill(eds), San Diego, doi:10.1016/B978-0-12-386941-8.00008-3, 1963.

Ziegler, H., An Introduction to Thermomechanics, North-Holland, 1983. 